\title{
RANKL coordinates multiple osteoclastogenic pathways by regulating expression of ubiquitin ligase RNF146
}

\author{
Yoshinori Matsumoto, ${ }^{1}$ Jose Larose, ${ }^{1}$ Oliver A. Kent, ${ }^{1}$ Melissa Lim, ${ }^{1}$ Adele Changoor, ${ }^{2}$ Lucia Zhang, ${ }^{2}$ Yaryna Storozhuk, ${ }^{2}$ \\ Xiaohong Mao, ${ }^{3}$ Marc D. Grynpas, ${ }^{2}$ Feng Cong, ${ }^{3}$ and Robert Rottapel ${ }^{1,4,5,6,7}$ \\ 1Princess Margaret Cancer Center, University Health Network, University of Toronto, Toronto, Ontario, Canada. ²unenfeld-Tanenbaum Research Institute, Mount Sinai Hospital, Toronto, Ontario, Canada. \\ ${ }^{3}$ Developmental and Molecular Pathways, Novartis Institute of Biomedical Research, Cambridge, Massachusetts, USA. ${ }^{4}$ Department of Medicine, ${ }^{5}$ Department of Medical Biophysics, and \\ ${ }^{6}$ Department of Immunology, University of Toronto, Toronto, Ontario, Canada. 'Division of Rheumatology, St. Michael's Hospital, Toronto, Ontario, Canada.
}

\begin{abstract}
Bone undergoes continuous remodeling due to balanced bone formation and resorption mediated by osteoblasts and osteoclasts, respectively. Osteoclasts arise from the macrophage lineage, and their differentiation is dependent on RANKL, a member of the TNF family of cytokines. Here, we have provided evidence that RANKL controls the expression of 3BP2, an adapter protein that is required for activation of SRC tyrosine kinase and simultaneously coordinates the attenuation of $\beta$-catenin, both of which are required to execute the osteoclast developmental program. We found that RANKL represses the transcription of the E3 ubiquitin ligase RNF146 through an NF- $\mathrm{BB}$-related inhibitory element in the RNF146 promoter. RANKL-mediated suppression of RNF146 results in the stabilization of its substrates, 3BP2 and AXIN1, which consequently triggers the activation of SRC and attenuates the expression of $\beta$-catenin, respectively. Depletion of RNF146 caused hypersensitivity to LPS-induced TNF- $\alpha$ production in vivo. RNF146 thus acts as an inhibitory switch to control osteoclastogenesis and cytokine production and may be a control point underlying the pathogenesis of chronic inflammatory diseases.
\end{abstract}

\section{Introduction}

RANKL, a member of the TNF superfamily of cytokines, is an obligate factor required for osteoclast differentiation and survival, lymph node organogenesis, lymphocyte development, and breast epithelial proliferation (1-6). Mice deficient in RANKL develop severe osteopetrosis due to low bone turnover as a result of absent functional osteoclasts (3). Inhibition of RANKL is now a major therapeutic modality in treating diseases of high bone turnover states, such as osteoporosis.

RANKL signals principally through the 2 transcription factors nuclear factor of activated T cells $\mathrm{c} 1$ (NFATc1) and NF- $\mathrm{KB}(2,3,7$, 8). In addition to the induction of the osteoclastogenic master transcription factor NFATc1 by RANKL signaling, osteoclast development requires modulation of 2 additional signaling pathways: the coordinated activation of the SRC tyrosine kinase $(9,10)$ with the simultaneous inhibition of the Wnt/ $\beta$-catenin pathway (11-13).

We previously reported that the adaptor protein $3 \mathrm{BP} 2$, which is encoded by the $S H 3 B P 2$ locus, is essential for normal osteoclast formation, since $\operatorname{Sh} 3 b p 2^{-/-}$mice show reduced bone resorption due to a cell-autonomous osteoclast defect (14). We have shown that 3BP2 nucleates a signaling complex, including SRC, VAV, and SYK, and that SRC activation is impaired in 3BP2-null osteoclasts. Constitutively active SRC rescues defective osteoclastogenesis

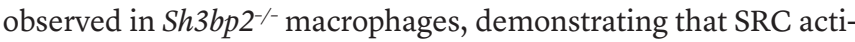
vation by $3 \mathrm{BP} 2$ is essential for normal osteoclast development.

Conflict of interest: The authors have declared that no conflict of interest exists

Submitted: September 6, 2016; Accepted: January 17, 2017.

Reference information: J Clin Invest. 2017;127(4):1303-1315

https://doi.org/10.1172/JCI90527.
3BP2 ligates the SRC SH3 domain, enforcing an open active configuration of SRC, and is both sufficient and necessary for SRC kinase activation in osteoclasts (14).

3PB2 is subject to tight negative regulation by tankyrase, a poly(ADP-ribose) polymerase (PARP) family member. Tankyrase-mediated 3BP2 ribosylation creates a recognition site for the E3 ubiquitin ligase RNF146, leading to its proteasomal degradation (15). Gain-of-function missense mutations in the SH3BP2 gene, which are associated with cherubism, a rare genetic syndrome characterized by bone dysplasia and remodeling of the facial bones (15-17), uncouple 3BP2 from tankyrase/RNF146, stabilize 3BP2 protein, and cause hyperactivation of SRC, SYK, and VAV. A genetically engineered mouse model of cherubism harboring one of the cherubism mutations develops hyperactive osteoclasts and a lethal systemic inflammatory disorder characterized by monocytic infiltration of visceral organs and elevated levels of TNF- $\alpha$ (18). Osteoclasts derived from the cherubism mice have increased endogenous 3BP2 protein levels and highly active SRC (15). 3BP2 is thus a concentration-limited adapter protein that controls SRC, VAV, and SYK activity in the monocyte lineage (15), yet little is currently known about how 3BP2 protein levels are controlled during osteoclastogenesis.

The Wnt/ $\beta$-catenin pathway antagonizes normal osteoclastogenesis. $\beta$-Catenin-deficient $\left(\mathrm{Ctnnb1}^{-/}\right)$mice display osteoporosis due to enhanced osteoclast differentiation, whereas mice harboring a constitutively active form of $\beta$-catenin are osteopetrotic due to defective osteoclastogenesis (11-13). $\beta$-Catenin is negatively regulated by a destruction complex that includes AXIN (19), gly-

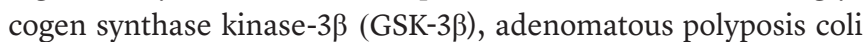
(APC), and the E3 ubiquitin ligase $\beta$-TrCP and leads to its phos- 
A
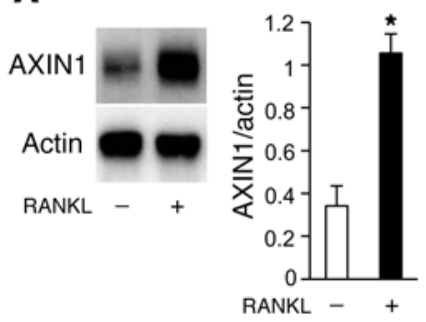

D

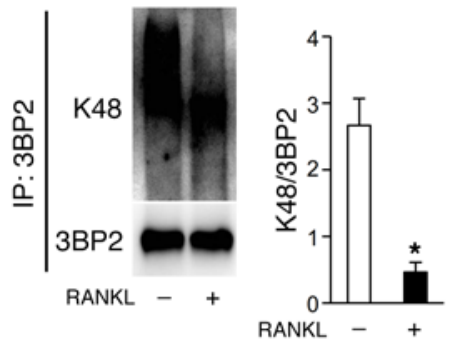

B

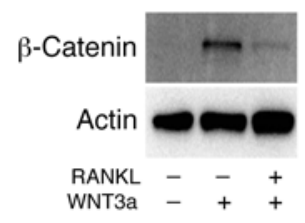

C

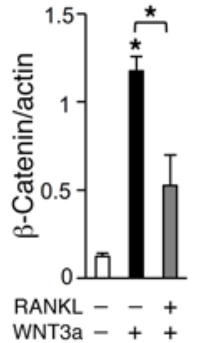

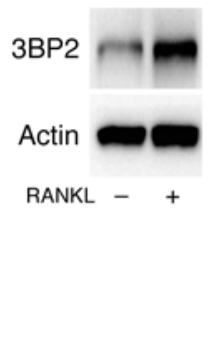

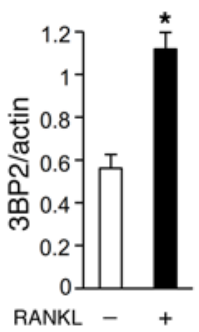

E

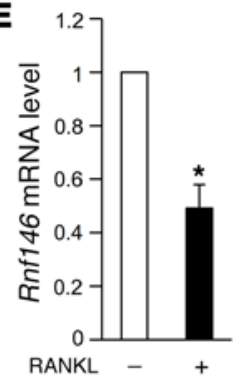

$\mathbf{F}$

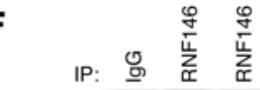

RNF146

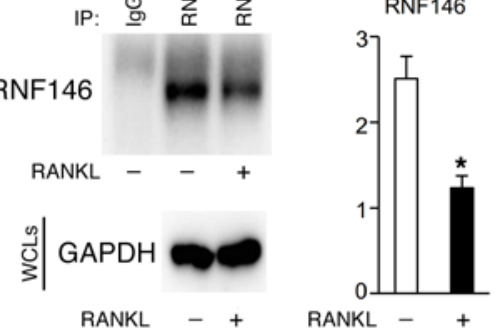

G

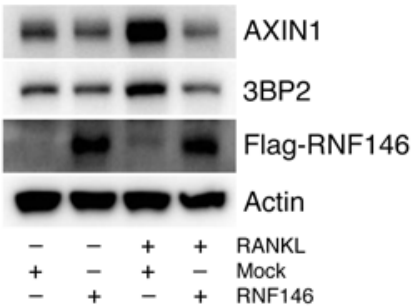

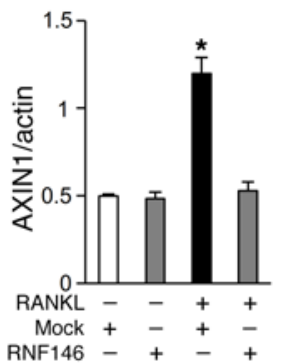

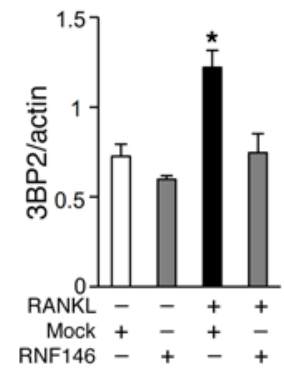

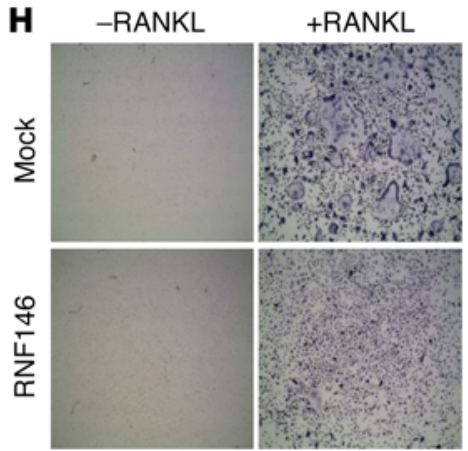

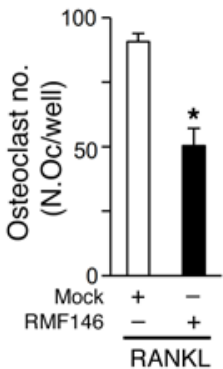

Figure 1. RANKL stabilizes 3BP2 and AXIN1 protein levels through suppression of the E3 ubiquitin ligase RNF146. (A) Primary murine macrophages were cultured in the presence or absence of RANKL $(50 \mathrm{ng} / \mathrm{ml}$ ). Whole cell lysates were probed with the indicated antibodies for Western blot analysis. (B) Primary murine macrophages were cultured in the presence or absence of RANKL $(50 \mathrm{ng} / \mathrm{ml})$ and a WNT3a-conditioned medium. Whole cell lysates were probed with the indicated antibodies for Western blot analysis. (C) Whole cell lysates from cells in A were probed with the indicated antibodies for Western blot analysis. (D) Primary murine macrophages cultured in the presence or absence of RANKL ( $50 \mathrm{ng} / \mathrm{ml})$ were treated with $10 \mu \mathrm{M}$ MG132 for 4 hours prior to collection of cell lysates. 3BP2 immune complexes were probed with an anti-K48 linkage-specific polyubiquitin or anti-3BP2 antibody. (E) qPCR analysis of Rnf146 mRNA expression in primary murine macrophages cultured in the presence or absence of RANKL $(50 \mathrm{ng} / \mathrm{ml})$. $n=3$. (F) Primary murine macrophages were cultured in the presence or absence of RANKL $(50 \mathrm{ng} / \mathrm{ml})$. RNF146 immune complexes were probed with an anti-RNF146 antibody. Whole cell lysates (WCLs) were probed with an anti-GAPDH antibody for Western blot analysis. (C) Primary murine macrophages infected with an empty vector control (mock) or RNF146-expressing retroviral vector were cultured in the presence or absence of RANKL (50 ng/ml). Whole cell lysates were probed with the indicated antibodies for Western blot analysis. (H) TRAP staining of osteoclasts infected with an empty vector control (mock) or RNF146-expressing retroviral vector and cultured in the presence or absence of RANKL for 7 days. Original magnification, $\times 40 . n=3$. $P$ values were determined by ANOVA with Tukey-Kramer's post hoc test (B and $\mathbf{G})$ or unpaired $t$ test $(\mathbf{A}, \mathbf{C}-\mathbf{F}$, and $\mathbf{H})$. Data are presented as mean \pm SEM. ${ }^{*} P<0.05$.

phorylation, ubiquitylation, and proteasome-dependent degradation (20). RNF146 targets AXIN for ubiquitylation and destruction and is thus a positive regulator of the Wnt $/ \beta$-catenin pathway (21). The molecular basis for repression of the Wnt/ $\beta$-catenin pathway during osteoclastogenesis is not known.

We show that RANKL simultaneously coordinates activation of SRC through induction of 3BP2 and suppression of $\beta$-catenin in addition to its established role in activating NFATc1. We demonstrate that RANKL represses the expression of the E3 ubiquitin ligase RNF146, which results in the stabilization of 3BP2 (15) and AXIN1 (21), leading to activation of SRC and suppression of $\beta$-catenin, respectively. We show that activation of NF-kB by RANKL signaling binds to an inhibitory element in the RNF146 promoter to potently repress RNF146 transcription. Finally, we found that dysfunction of the 3BP2 degradation program controlled by RNF146 in mice results in accelerated osteoclastogenesis and hypersensitivity to LPS induction of TNF- $\alpha$ in macrophages.

Our study has unveiled a pathway downstream of RANKL that links bone resorption to inflammatory states, a condition that underlies human diseases, such as rheumatoid arthritis.

\section{Results}

RANKL stabilizes $3 B P 2$ and AXIN1 protein levels through suppression of the E3 ubiquitin ligase RNF146. The 3BP2/SRC (14), Wnt/ $\beta$-cat- 

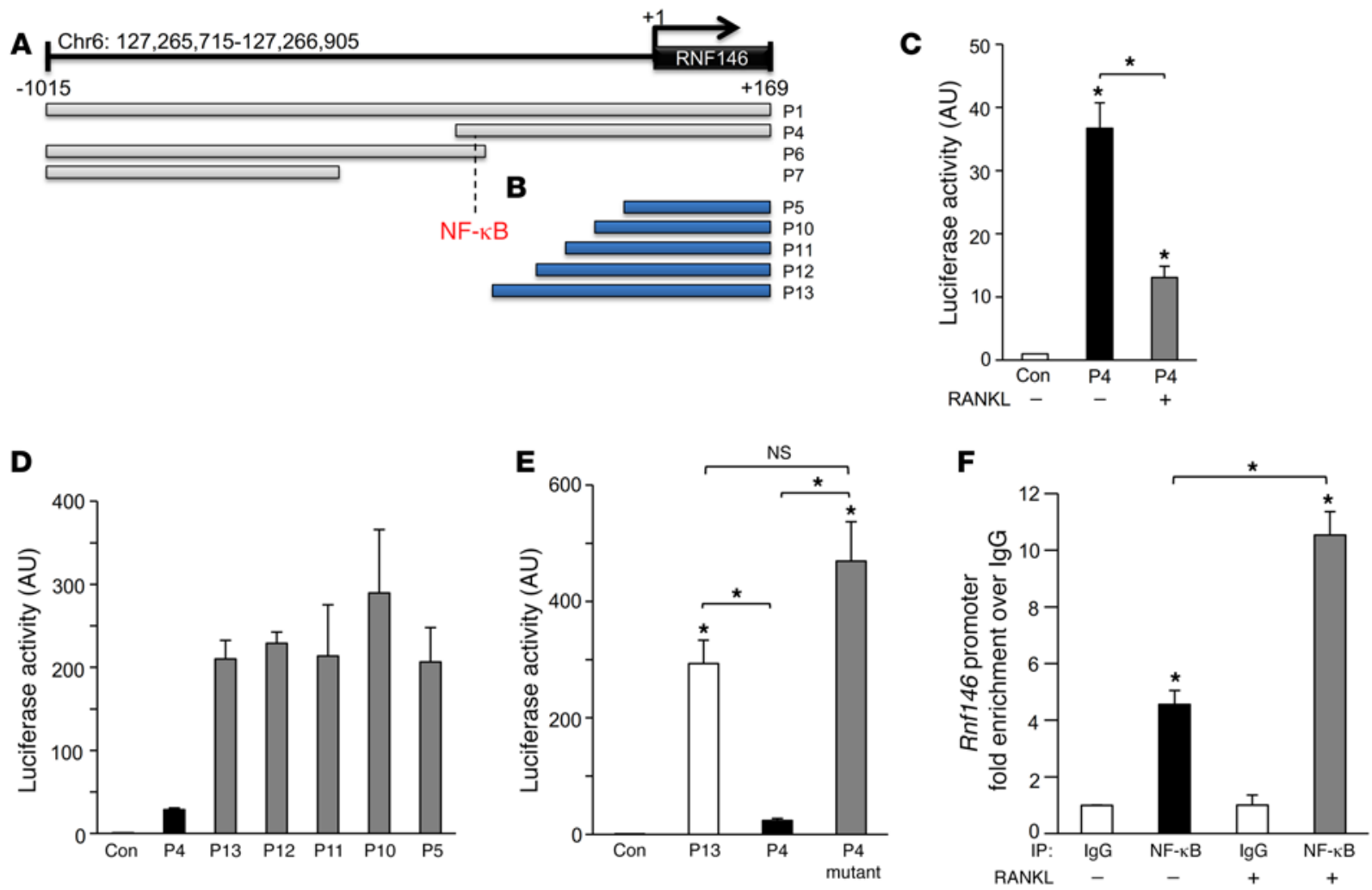

Figure 2. RANKL suppresses RNF146 promoter activity through activation of NF-KB. (A and B) Boundaries of RNF146 promoter constructs cloned into the pCL3-basic luciferase reporter vector. Numbers are relative to the transcription start site of the major RefSeq (RefSeq Accession: NM_030963) transcript. Dashed line indicates a potential binding site for NF-кB between P4 and P13. (C) Luciferase activity from the indicated RNF146 promoter constructs transfected in RAW264.7 cells. Cells were cultured in the presence or absence of RANKL $(100 \mathrm{ng} / \mathrm{ml}) . n=3$. (D and E) Luciferase activity from the indicated RNF146 promoter constructs transfected in RAW264.7 cells. $n=3$. (F) qPCR of chromatin immunoprecipitates from RAW264.7 cells cultured in the presence or absence of RANKL $(100 \mathrm{ng} / \mathrm{ml})$. Amplicons flank the NF- $\mathrm{KB}$-binding site within the Rnf146 promoter. Fold enrichment represents the signal obtained after immunoprecipitation with the indicated antibody over the nonspecific IgG antibody. $n=3$. $P$ values were determined by ANOVA with Tukey-Kramer's post hoc test (C, E, and $\mathbf{F})$. Data are presented as mean \pm SEM. ${ }^{*} P<0.05$.

enin (11-13), and RANKL/RANK $(2,3,7,8)$ pathways are all essential for normal osteoclast differentiation. We conjectured that these 3 pathways could be part of a common integrated signaling pathway during osteoclastogenesis. We first examined the effect of RANKL on levels of AXIN1, a negative regulator of the Wnt/ $\beta$-catenin pathway, and observed that AXIN1 protein expression was elevated 3-fold by RANKL in primary murine macrophages without enhancement of Axin1 transcripts (Figure 1A and Supplemental Figure 1A; supplemental material available online with this article; https://doi.org/10.1172/JCI90527DS1). Additionally, RANKL impaired WNT3a-induced stabilization of $\beta$-catenin (Figure 1B), suggesting that RANKL-mediated AXIN1 elevation may be functionally coupled to the control of $\beta$-catenin. We next examined the effect of RANKL on 3BP2 levels and observed that RANKL also enhanced 3BP2 protein expression 2-fold in primary murine macrophages (Figure 1C) without elevation of the Sh3bp2 transcript levels (Supplemental Figure 1B). These data suggest that both 3BP2 and AXIN1 proteins are likely stabilized through a posttranscriptional mechanism downstream of RANK signaling. In the previous studies, we and others showed that 3BP2 and AXIN1 are negatively regulated through tankyrase-mediated ADP ribosylation, which creates a recognition-binding site for the E3 ubiquitin ligase RNF146 $(15,20,21)$. We observed that RANKL suppressed lysine 48 ubiquitylation of 3BP2 without changes in the levels of ADP ribosylation in primary murine macrophages (Figure 1D and Supplemental Figure 1C). These results suggest that the attenuated levels of 3BP2 ubiquitylation could be the result of decreased RNF146 expression, activity, or both. Consistent with these results, we found that expression levels of Rnf146 mRNA and protein were reduced in RANKL-treated macrophages (Figure 1, E and F).

These data suggest that RANKL-induced osteoclastogenesis may in part be mediated by the coordinated stabilization of 3BP2 and suppression of $\beta$-catenin, a known inhibitor of osteoclast formation (11-13), through the repression of RNF146. To determine whether the stabilization of 3BP2 and AXIN1 by RANKL is achieved specifically through repression of Rnfl46 transcription, we sought to rescue this effect by ectopic expression of RNF146. We infected primary murine macrophages with an RNF146expressing retroviral vector and observed that ectopic expression of RNF146 restored the 3BP2 and AXIN1 expression levels elevated by RANKL to normal levels and impaired osteoclast differentiation (Figure 1, G and $\mathrm{H}$ ). These findings demonstrate that RANKL stabilizes 3BP2 and AXIN1 through suppression of the E3 ubiquitin ligase RNF146, leading to enhanced osteoclastogenesis.

RANKL suppresses RNF146 promoter activity through activation of NF- $\kappa B$. To elucidate the basis of RANKL transcriptional repres- 

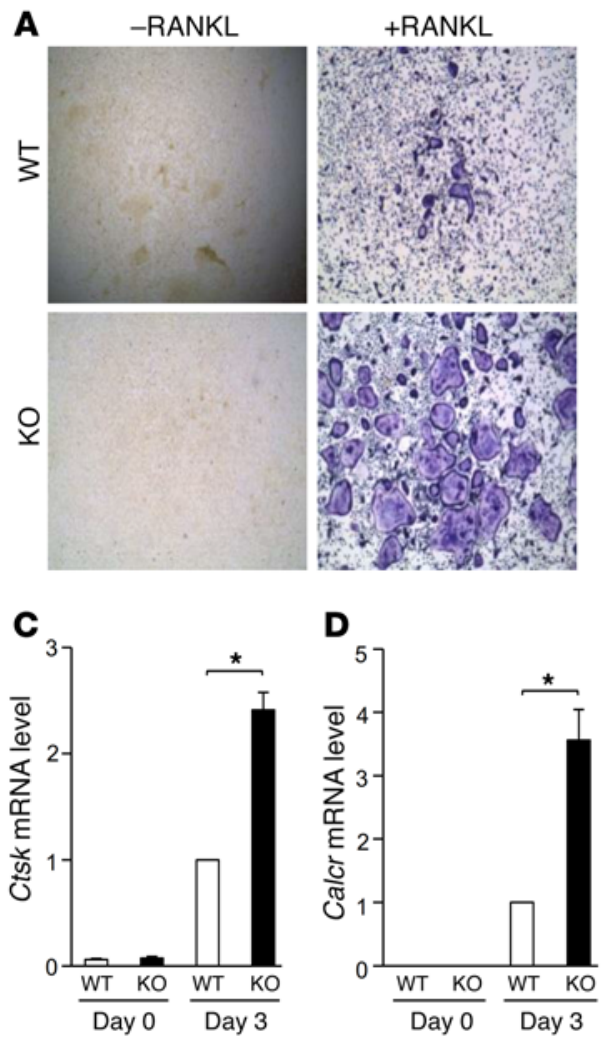

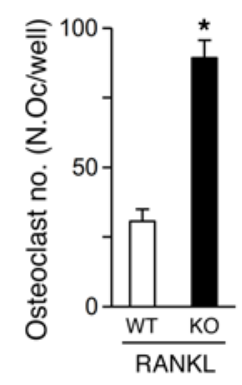

B
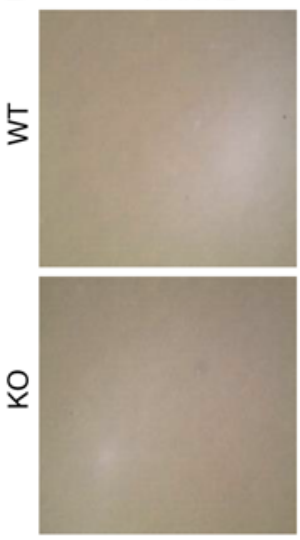

E

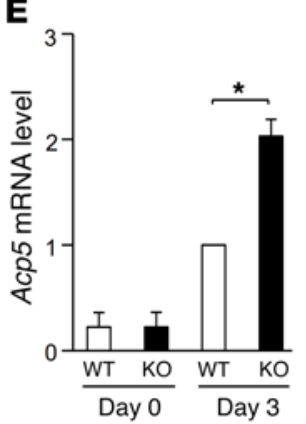

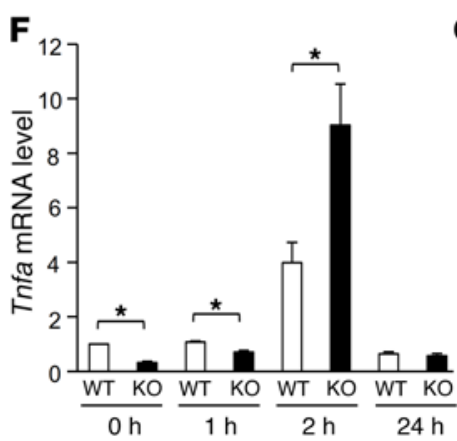

+RANKL
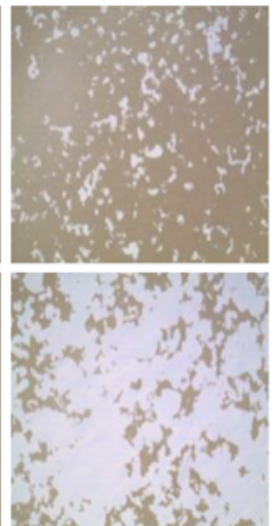

G

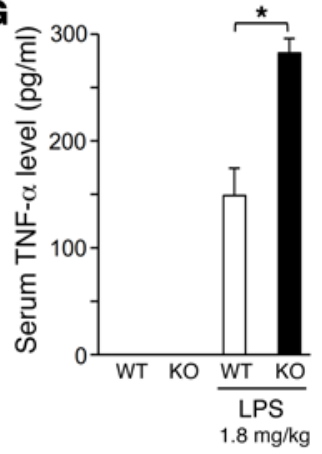

Figure 3. RNF146 represses osteoclastogenesis and LPS-mediated TNF- $\alpha$ production in bone marrow-derived osteoclast progenitors. (A and B) TRAP staining (A) or resorption pit assay (B) of osteoclasts derived from WT and Rnf146 $6^{f / f l}$ LysM-Cre (KO) osteoclast progenitors cultured in the presence or absence of RANKL for 7 days. Original magnification, $\times 40 . n=3$. (C-E) qPCR analysis of Ctsk (C), Calcr (D), and Acp5 (E) mRNA expression in primary murine osteoclast progenitors derived from WT and Rnf146 $6^{f / f l}$ LysM-Cre (KO) mice and cultured in the presence of RANKL for 0 to 3 days. The relative expression of each mRNA was normalized to that in WT cells at day 3. $n=3$. (F) qPCR analysis of $T n f \alpha$ mRNA expression in primary murine osteoclast progenitors derived from WT and Rnf146 $6^{f / f l}$ LysM-Cre (KO) mice and cultured in the presence of LPS (10 ng/ml) for 0 to 24 hours. $n=3$. (G) WT and Rnf146 $6^{f / f l}$ LysM-Cre (KO) mice were injected i.p. with LPS, and TNF- $\alpha$ in the serum was measured by ELISA 4 hours later. $n=5$. $P$ values were determined by unpaired $t$ test. Data are presented as mean $\pm \mathrm{SEM}$. ${ }^{*} P<0.05$.

sion of RNF146, we performed functional analysis of RNF146 promoter cloned into a luciferase reporter construct. We generated 9 constructs derived from a 1-kb RNF146 promoter fragment upstream of the putative transcriptional start site (Figure 2, A and $\mathrm{B})$. We observed robust luciferase activity from $\mathrm{P} 1$ and $\mathrm{P} 4$ promoters, but not from P6 and P7 promoters, in 293T cells, demonstrating that $\mathrm{P} 1$ and $\mathrm{P} 4$ are active promoter fragments (Figure $2 \mathrm{~A}$ and Supplemental Figure 2A). We next examined the promoter activities in response to RANKL stimulation and observed that RANKL strongly inhibited P4 luciferase activity in a murine macrophage cell line, RAW264.7 (Figure 2C). To identify the minimal inhibitory promoter elements in $\mathrm{P} 4$ that were responsive to RANKL treatment, we cloned a series of truncated promoter fragments, shown in Figure 2B. Promoter activity derived from all of the smaller fragments was 7 times higher than that of P4 (Figure 2D), suggesting the presence of a negative regulatory element between P4 and P13 (Figure 2, A and B). To determine the transcription factors that could potentially bind to the negative regulatory region, we analyzed the sequence of P4 that was absent from P13 using MATCH, a transcription factor-binding prediction algorithm (22), and found a conserved sequence within the RNF146 promoter that corresponded to a potential binding site for NF- $\kappa \mathrm{B}$ (Figure
2, A and B, and Supplemental Figure 2, B and C). To evaluate the functional relevance of this sequence, we introduced mutations in the putative NF- $\kappa \mathrm{B}$-binding site in the $\mathrm{P} 4$ promoter fragment to disrupt NF- $\kappa \mathrm{B}$ binding (P4 mutant), as predicted by the position weight matrix (Supplemental Figure 2C), and observed that the P4 mutant construct augmented luciferase activity to levels similar to those of promoter P13 (Figure 2E) and that neither P4 mutant nor P13 promoter activity was suppressed by RANKL (Supplemental Figure 2D). To confirm that NF- $\kappa \mathrm{B}$ regulates endogenous $R n f 146$ transcription, we performed ChIP of the Rnf146 promoter in RAW264.7 cells and observed strong enrichment of an Rnf146derived amplicon in NF- $\kappa \mathrm{B}$ chromatin immunoprecipitates (Figure $2 \mathrm{~F}$ ). Importantly, we found that recruitment of NF- $\mathrm{KB}$ to the Rnf146 promoter was increased 2-fold by RANKL treatment (Figure $2 \mathrm{~F}$ ). These results are consistent with a model whereby RANKL stabilizes 3BP2 and AXIN1 via NF- $\mathrm{B}$ transcriptional repression of $R N F 146$, leading to enhanced osteoclast differentiation.

RNF146 represses osteoclastogenesis and LPS-mediated TNF- $\alpha$ production in bone marrow-derived osteoclast progenitors. To test the hypothesis that RANKL-mediated repression of RNF146 coordinates enhanced 3BP2 signaling with the attenuated $\beta$-catenin in osteoclasts, we generated Rnf146 conditional knockout mice 
A

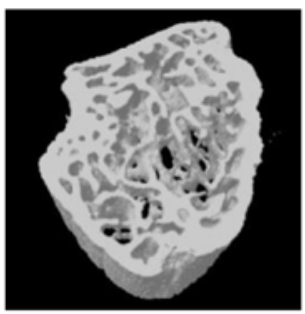

Rnf146 $6^{\text {III }}$ LysM-Cre (KO)
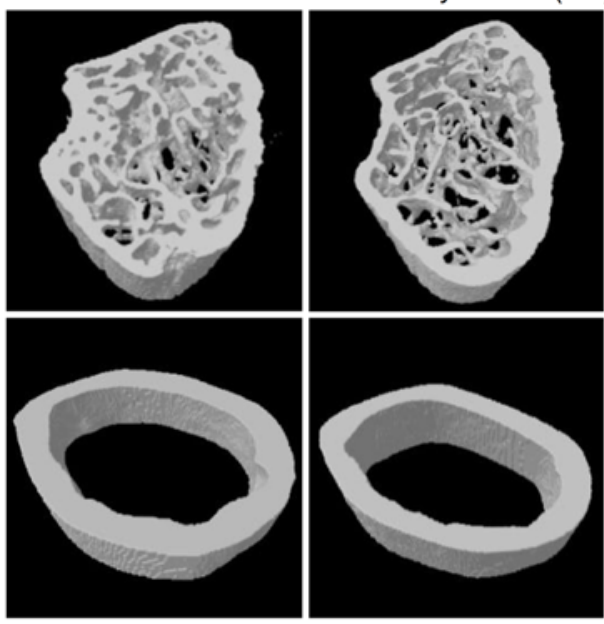

H

WT

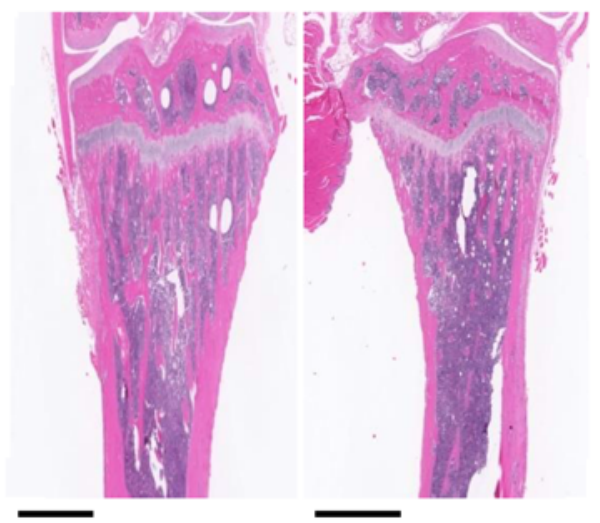

$\mathbf{L}$

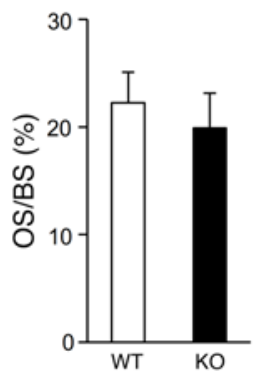

KO

B

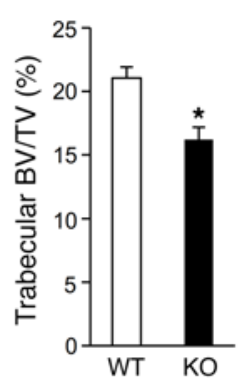

E

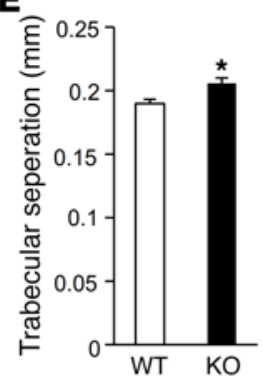

I

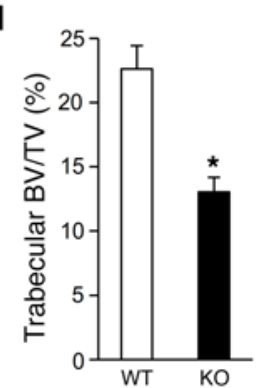

N

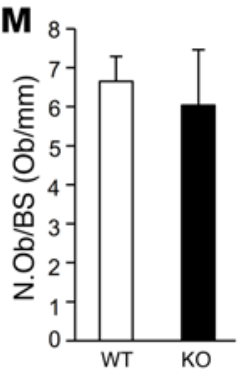

C

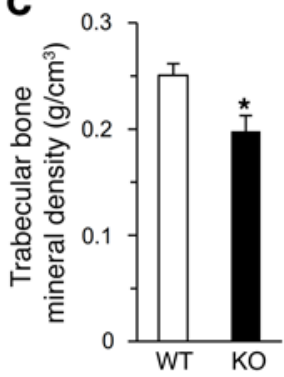

$\mathbf{F}$

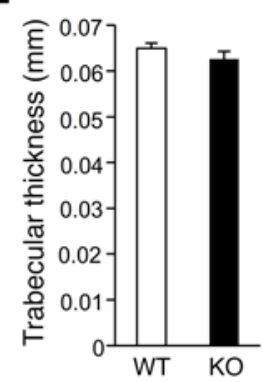

J

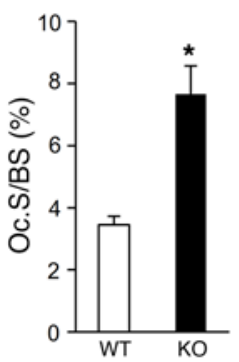

$\mathbf{0}$

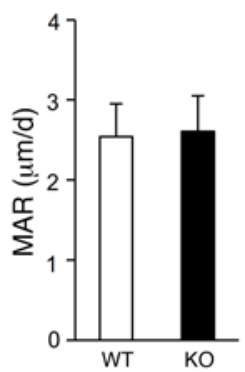

D

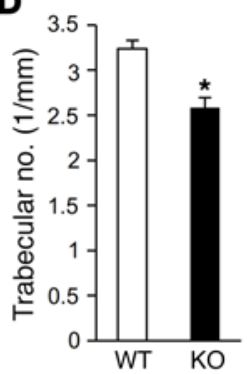

G

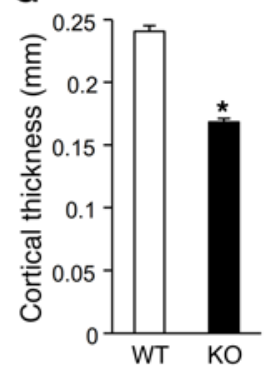

$\mathbf{K}$
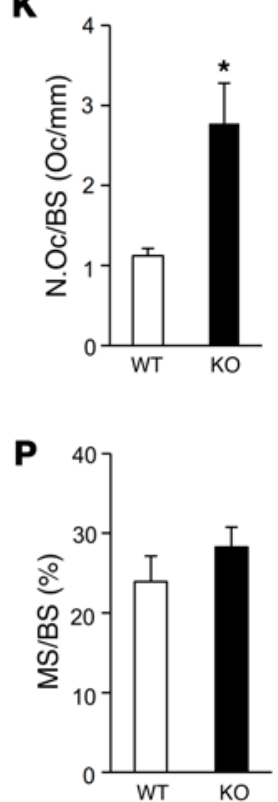

Figure 4. Rnf146 ${ }^{f / f l}$ LysM-Cre mice are osteopenic due to active osteoclastogenesis. (A) $\mu C T$ reconstruction of the trabecular region below the distal femur growth plate (top panels) and the cortical region of midshaft femurs (bottom panels) of 12-week-old WT and Rnf146 fl/fl LysM-Cre (KO) mice. (B-C) $\mu \mathrm{CT}$-derived measurements of trabecular bone volume per total volume (BV/TV) (B), trabecular bone mineral density (C), trabecular number (D), trabecular separation (E), trabecular thickness (F), and cortical thickness (C) of 12-week-old WT and Rnf146 fl/ff LysM-Cre (KO) mice. $n=6$. (H) H\&E staining of tibiae from 12-week-old WT and Rnf146 fl/fl LysM-Cre (KO) mice. Scale bars: $1 \mathrm{~mm}$. (I-M) Histomorphometric analysis of trabecular bone volume per total volume (I), osteoclast surface per bone surface (Oc.S/BS) (J), osteoclast number per bone surface (N.Oc/BS) (K), osteoid surface per bone surface (OS/BS) (L), and osteoblast number per bone surface (N.Ob/BS) (M) of 12-week-old WT and Rnf146 $6^{f l / f l}$ LysM-Cre (KO) mice. $n=5-7$. (N-P) Dynamic histomorphometric analysis of tibial trabecular bone formation rate (BFR/BS) (N), mineral apposition rate (MAR) (0), and mineralizing surface (MS/BS) (P) of 12-week-old WT and $R n f 146^{f / f f} L y s M-C r e(K O)$ mice. $n=5-6$. $P$ values were determined by unpaired $t$ test. Data are presented as mean $\pm \mathrm{SEM}$. ${ }^{*} P<0.05$.

( $\left.R n f 146^{f / f l} L y s M-C r e\right)$ to examine the in vivo bone phenotype in the absence of RNF146 within the macrophage compartment (Supplemental Figure 3, A-D). We observed that excision of RNF146 in osteoclast progenitors accelerated in vitro osteoclast differentiation and enhanced bone resorptive capacity in response to macrophage CSF (M-CSF) and RANKL compared with WT cells (Figure 3, A and B). The capacity of Rnf146 $6^{f / f l} L y s M$-Cre osteoclast progen- itors to execute the osteoclastogenic differentiation program, as assessed by the induction of Ctsk, Calcr, and Acp5 mRNAs, was significantly enhanced compared with that of WT cells (Figure 3, C-E). In addition to the developmental effects of loss of RNF146 on osteoclastogenesis, we found that Tnf $\alpha$ mRNA expression in response to a TLR4 ligand, LPS, was enhanced in Rnf146 $6^{f / f l} L y s M-$ Cre progenitors compared with that in WT cells (Figure 3F). More- 
A
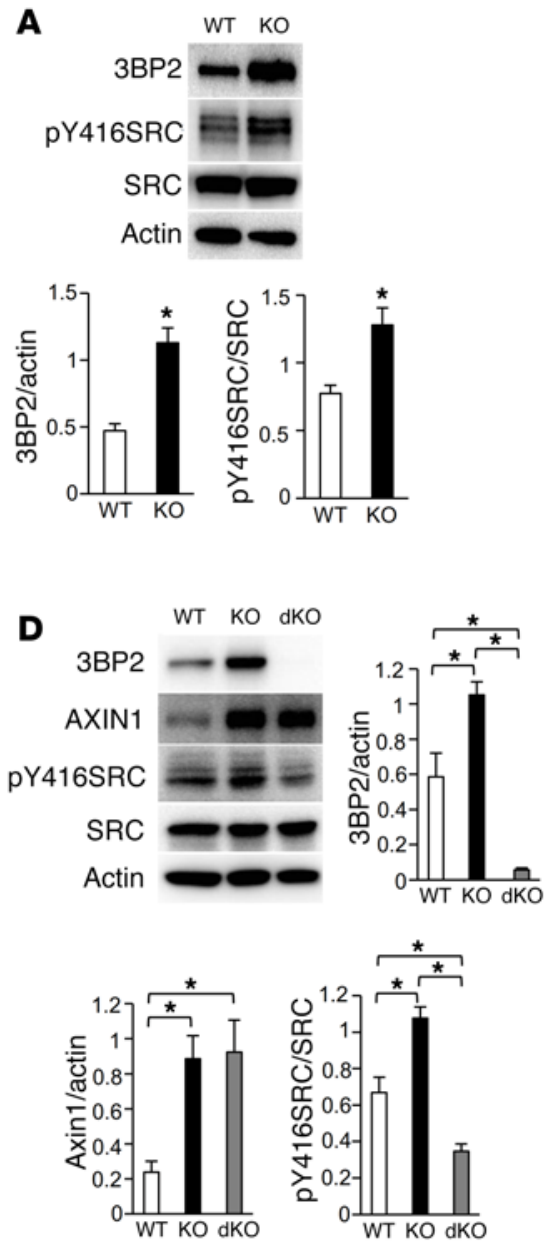

B
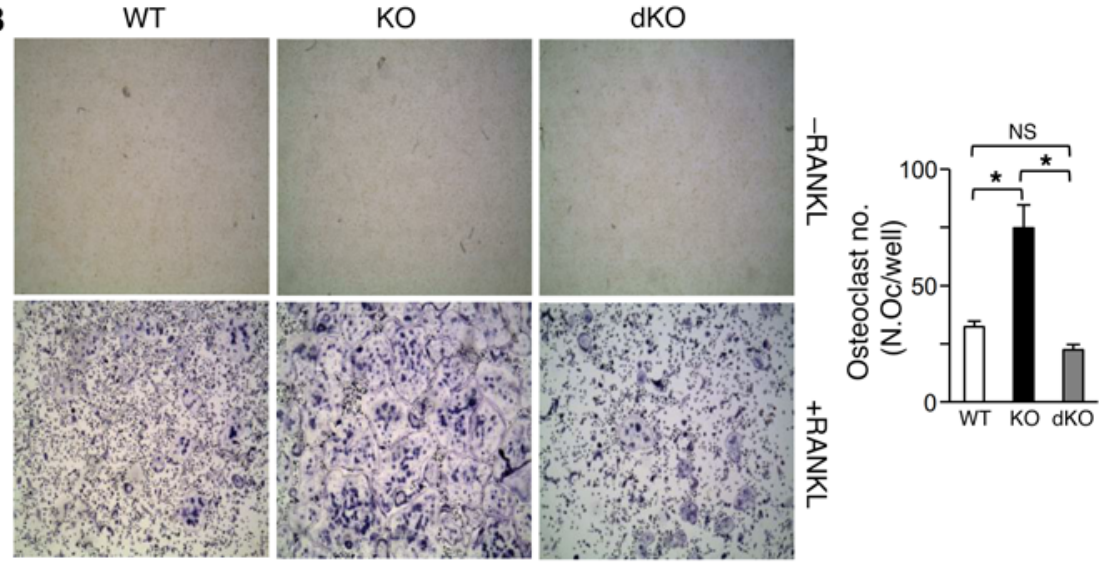

E

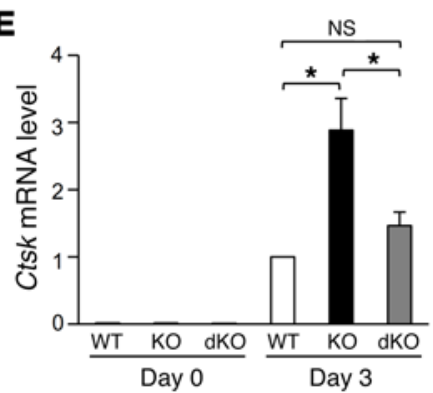

C
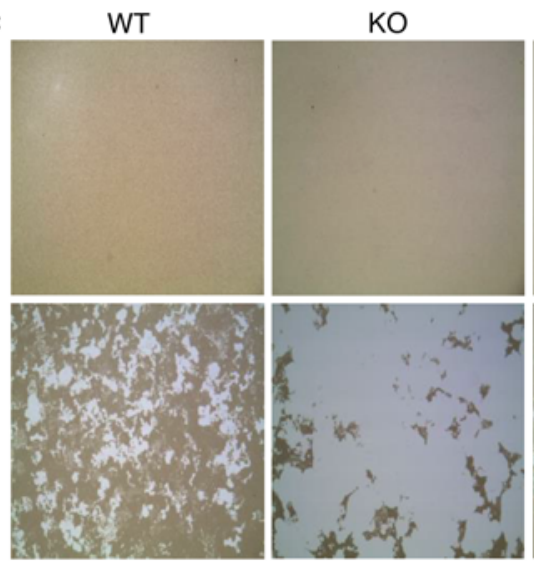

dKO
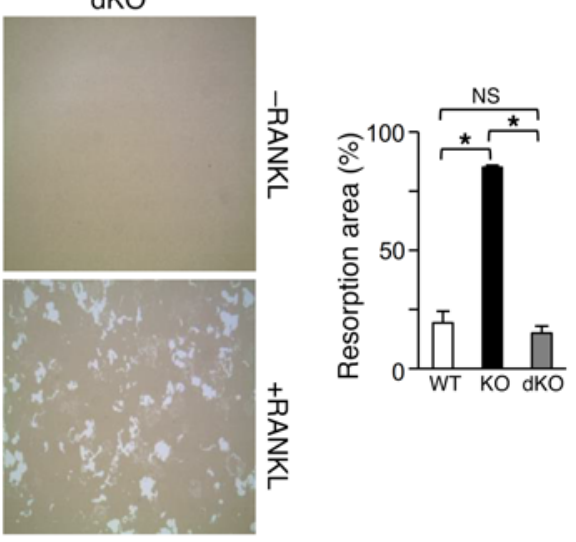

H

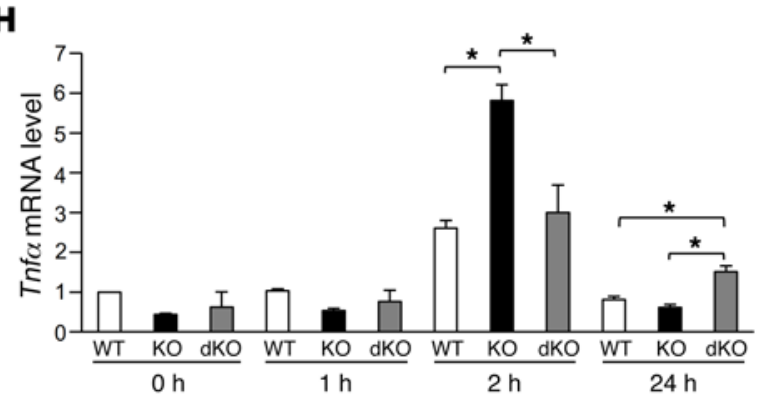

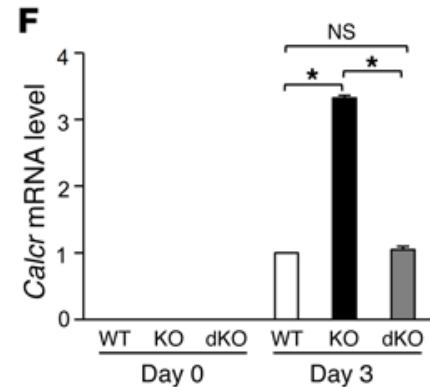
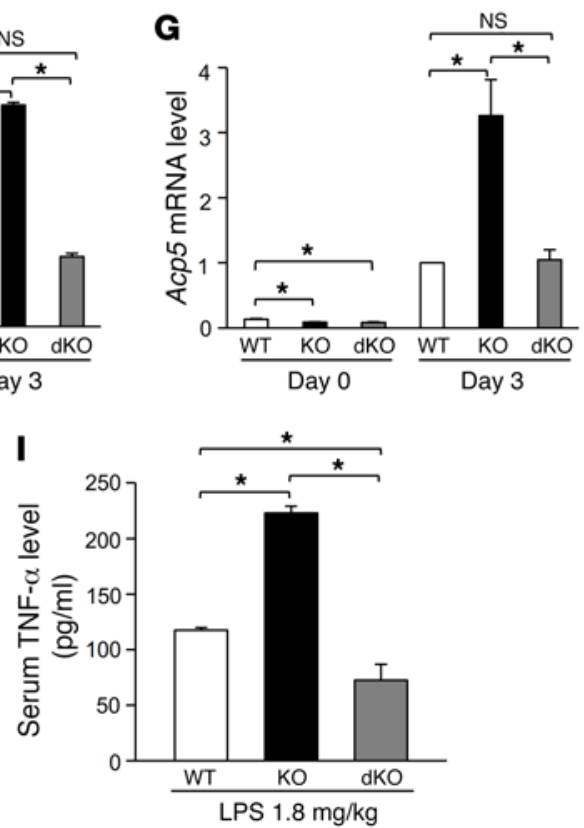
Figure 5. Stabilization of 3BP2 is required for activated osteoclasts and TNF- $\alpha$ production in Rnf146 $6^{f / f l}$ LysM-Cre osteoclast progenitors. (A) Whole cell lysates from primary murine osteoclast progenitors derived from WT and Rnf146 fl/fl LysM-Cre (KO) mice were probed with the indicated antibodies for Western blot analysis. (B and C) TRAP staining (B) or resorption pit assay (C) of osteoclasts derived from WT, Rnf146 $6^{f / f l}$ LysM-Cre (KO), and $R n f 146^{f l / f l} S h 3 b p 2^{f / f l} L y s M$-Cre (dKO) osteoclast progenitors cultured in the presence or absence of RANKL for 7 days. Original magnification, $\times 40$. $n=3$. (D) Whole cell lysates from cells in $\mathbf{B}$ were probed with the indicated antibodies for Western blot analysis. (E-G) qPCR analysis of Ctsk (E), Calcr $(\mathbf{F})$, and $A c p 5(\mathbf{G})$ mRNA expression in primary murine osteoclast progeni-

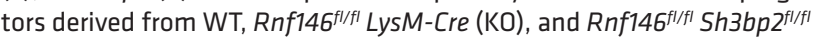
LysM-Cre (dKO) mice and cultured in the presence of RANKL for 0 to 3 days. The relative expression of each mRNA was normalized to that in WT cells at day 3. $n=3$. (H) qPCR analysis of Tnf $\alpha$ mRNA expression in primary murine osteoclast progenitors derived from WT, Rnf146 fl/fl LysM-Cre (KO), and $R n f 146^{f / f l} S h 3 b p 2^{f / f f l} L y s M-C r e$ (dKO) mice and cultured in the presence of LPS (10 ng/ml) for 0 to 24 hours. $n=3$. (I) WT, Rnf146 fl/fl LysM-Cre (KO), and $R n f 146^{f / f l} S h 3 b p 2^{f / / f l}$ LysM-Cre (dKO) mice were injected i.p. with LPS, and TNF- $\alpha$ in the serum was measured by ELISA 4 hours later. $n=6$. $P$ values were determined by ANOVA with Tukey-Kramer's post hoc test (B-I) or unpaired $t$ test $(\mathbf{A})$. Data are presented as mean \pm SEM. ${ }^{*} P<0.05$.

over, LPS-mediated TNF- $\alpha$ production in vivo was elevated in Rnf146 $6^{\text {A/f }} \mathrm{LysM}$-Cre mice compared with that in WT mice (Figure $3 \mathrm{G})$. These findings demonstrate that repression of RNF146 is a critical checkpoint during osteoclast maturation and LPS-induced TNF- $\alpha$ production in bone marrow-derived osteoclast progenitors.

$R n f 146^{A / f l} \mathrm{LysM}$-Cre mice are osteopenic due to active osteoclastogenesis. To investigate the role of RNF146 in skeletal remodeling, we analyzed the bones of Rnf146 $6^{A / f} \mathrm{LysM}$-Cre mice. 3D reconstruction of femora using microcomputed tomography $(\mu \mathrm{CT})$ showed osteopenia (Figure $4 \mathrm{~A}$ ), with a reduction of trabecular bone volume (Figure $4 \mathrm{~B}$ ), trabecular bone mineral density (Figure 4C), and trabecular number (Figure 4D), and an increased trabecular separation (Figure 4E) in Rnf146 $6^{\pi / f} \mathrm{LysM}$-Cre mice compared with that in WT mice, though trabecular thickness was under normal (Figure 4F). Additionally, a reduction of cortical thickness, cortical major and minor diameter, and cortical periosteal and endos-

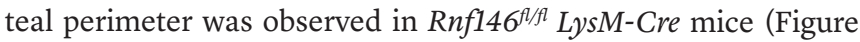
$4 \mathrm{G}$ and Supplemental Figure 4, A-D).

Histological analysis of tibiae from Rnf146 $6^{\nexists f l} L y s M$-Cre mice revealed low trabecular bone mass compared with that in WT mice (Figure $4, \mathrm{H}$ and I), consistent with the results of $\mu \mathrm{CT}$ analysis shown in Figure 4, A and B. To determine the cause of low

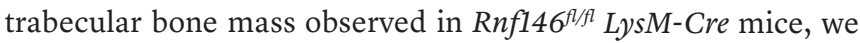
first analyzed osteoclasts in tibiae stained for tartrate-resistant acid phosphatase (TRAP) activity. Histomorphometric analysis showed increased osteoclast surface per bone surface and osteoclast number per bone surface in Rnf146 $6^{\pi / f l} \mathrm{LysM}$-Cre mice (Figure 4 , $\mathrm{J}$ and $\mathrm{K}$ ), indicating enhanced osteoclastogenesis. In contrast, trichrome staining of tibiae showed that osteoid surface per bone surface (Figure $4 \mathrm{~L}$ ) and osteoblast number per bone surface (Figure 4M) were similar in WT and Rnf146 ${ }^{f / f l} L y s M$-Cre mice. Moreover, dynamic bone histomorphometric analysis after calcein green labeling revealed that bone formation rate (Figure $4 \mathrm{~N}$ ), mineral apposition rate (Figure 4O), and mineralizing surface (Figure $4 \mathrm{P}$ ) were similar in WT and Rnf146 $6^{f / f l} L y s M$-Cre mice. These results demonstrate that $R n f 146^{f / f l} L y s M$-Cre mice show low bone mass due to enhanced osteoclastogenesis, but not defective osteoblastogenesis.

Stabilization of $3 B P 2$ is required for activated osteoclasts and TNF- $\alpha$ production in Rnf146 $6^{f / f} \mathrm{LysM}$-Cre osteoclast progenitors. We next investigated the molecular mechanisms underlying osteopenia resulting from activated osteoclasts in $R n f 146^{\ell / / l} \mathrm{~L} y s \mathrm{M}$-Cre mice. Given the role of the 3BP2/SRC pathway in promoting osteoclast maturation, we first examined the expression levels of 3BP2 in Rnf146 $6^{f / f l} \mathrm{LysM}$-Cre bone marrow-derived osteoclast progenitors and observed increased 3BP2 protein expression in these cells compared with that in WT cells, leading to activation of SRC kinase (Figure 5A). We queried whether 3BP2 is required to support the emergence of highly active osteoclasts observed in $R n f 146^{\Re / l l} L y s M$-Cre mice and generated Sh3bp2 conditional knockout mice (Sh3bp $\left.2^{f / f l} L y s M-C r e\right)$ in which 3BP2 was deleted in osteoclast progenitors (Supplemental Figure 5, A and B). We observed that deletion of Sh3bp2 in osteoclast progenitors suppressed in vitro osteoclast differentiation and bone resorptive capacity compared with WT cells with a reduction of active SRC levels (Supplemental Figure 5, C-E), consistent with our previous study (14). To confirm that deletion of Sh3bp2 could rescue the phonotype observed in $R n f 146^{f / f l} L y s M$-Cre mice, we next generated Rnfl46 and Sh3bp2 conditional double-knockout mice $\left(R n f 146^{f / f l} S h 3 b p 2^{f / f l} L y s M-C r e\right)$ in which both RNF146 and 3BP2 were deleted in osteoclast progenitors. As shown in Figure 5, B and $\mathrm{C}$, the highly activated osteoclast phenotype observed in $R n f 146^{f / l} L y s M$-Creprogenitorswasreversed in $R n f 146^{f / f} S h 3 b p 2^{f / f l}$ LysM-Cre cells. Consistent with this result, the activation of

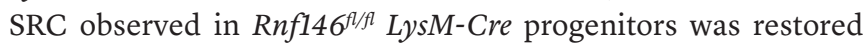
to near normal levels in Rnfl46 $6^{f / f l} S h 3 b p 2^{f / f l} L y s M$-Cre cells (Figure 5D). Additionally, the expression levels of Ctsk, Calcr, and Acp 5 mRNAs elevated in Rnf146 $6^{f / l} L y s M$-Cre progenitors were restored to normal in Rnf146 $6^{A / f} S h 3 b p 2^{f / f l} L y s M$-Cre cells (Figure 5, E-G). Finally, we observed that the hypersensitive response to LPS-induced TNF- $\alpha$ production in Rnf14 $6^{\nexists f l} \mathrm{LysM}$-Cre mice was ameliorated in Rnf146 $6^{f / f l} S h 3 b p 2^{f / f l} L y s M$-Cre mice (Figure 5, H and I). These results indicate that stabilization of 3BP2 protein is required for osteoclast activation and hypersensitivity to LPS in Rnf146 $6^{f / f l} L y s M-C r e$ mice.

Loss of $3 B P 2$ rescued osteopenia observed in Rnf146/f/l LysM-Cre mice. We next determined whether the severe osteopenic phe-

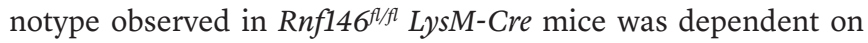
3BP2. Consistent with our previous study (14), Sh3bp $2^{f / f l} L y s M-C r e$ mice showed increased bone mass (Figure 6A), with an elevation of trabecular bone volume (Supplemental Figure 6A), trabecular bone mineral density (Supplemental Figure 6B), trabecular number (Supplemental Figure 6C), and trabecular thickness (Supplemental Figure 6D) compared with WT mice, though trabecular separation was near normal (Supplemental Figure 6E). Cortical thickness, cortical major diameter, and cortical periosteal perimeter, but not cortical minor diameter and cortical endosteal perim-

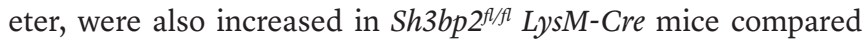
with WT mice (Supplemental Figure 6, F-J). In concert with the data shown in Supplemental Figure 5, C-E, these results demonstrate that 3BP2 expression in osteoclast progenitors is required for normal osteoclastogenesis and bone resorption to maintain bone mass. We finally confirmed that osteopenia observed in $R n f 146^{\text {fl/l }}$ 
A
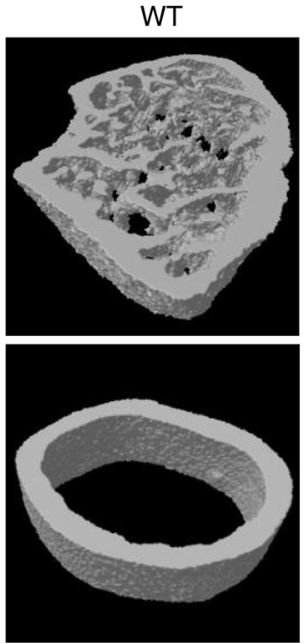

C

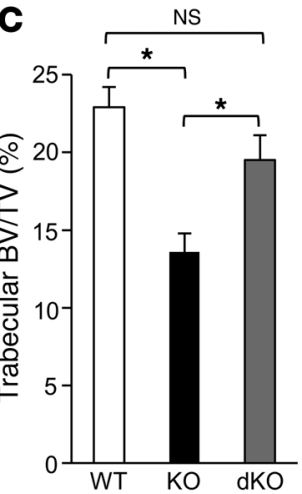

D

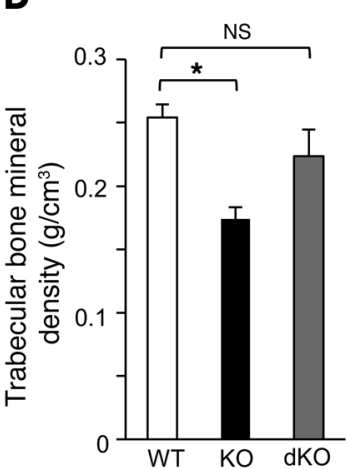

Sh3bp2 fl/fl LysM-Cre
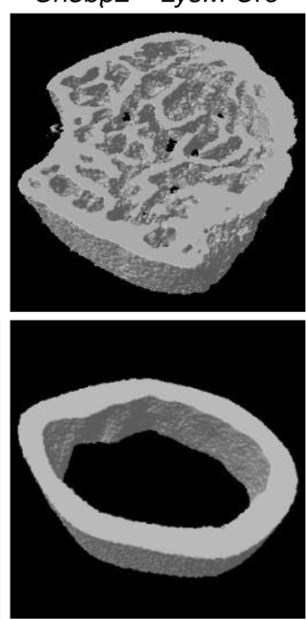

B
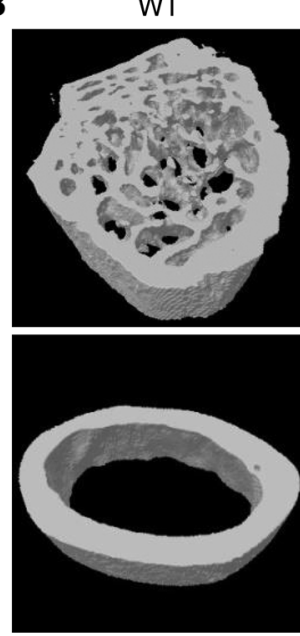

E

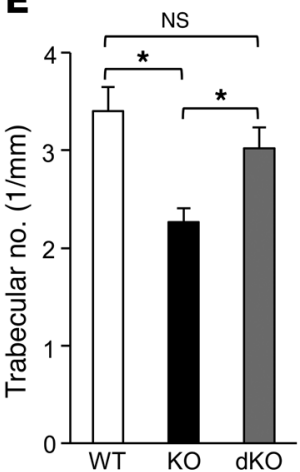

Rnf146

LysM-Cre (KO)
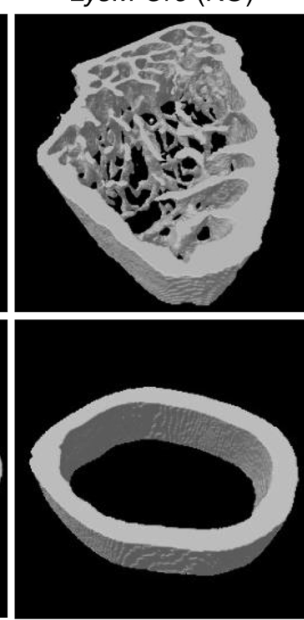

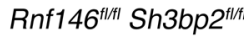

LysM-Cre (KO)

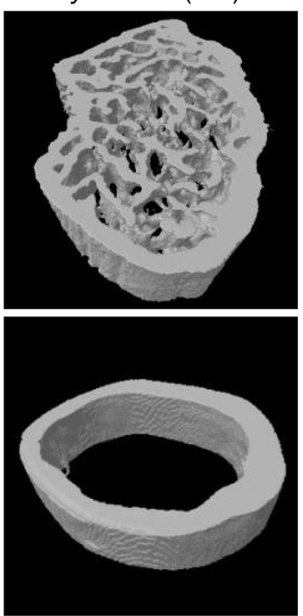

$\mathbf{F}$
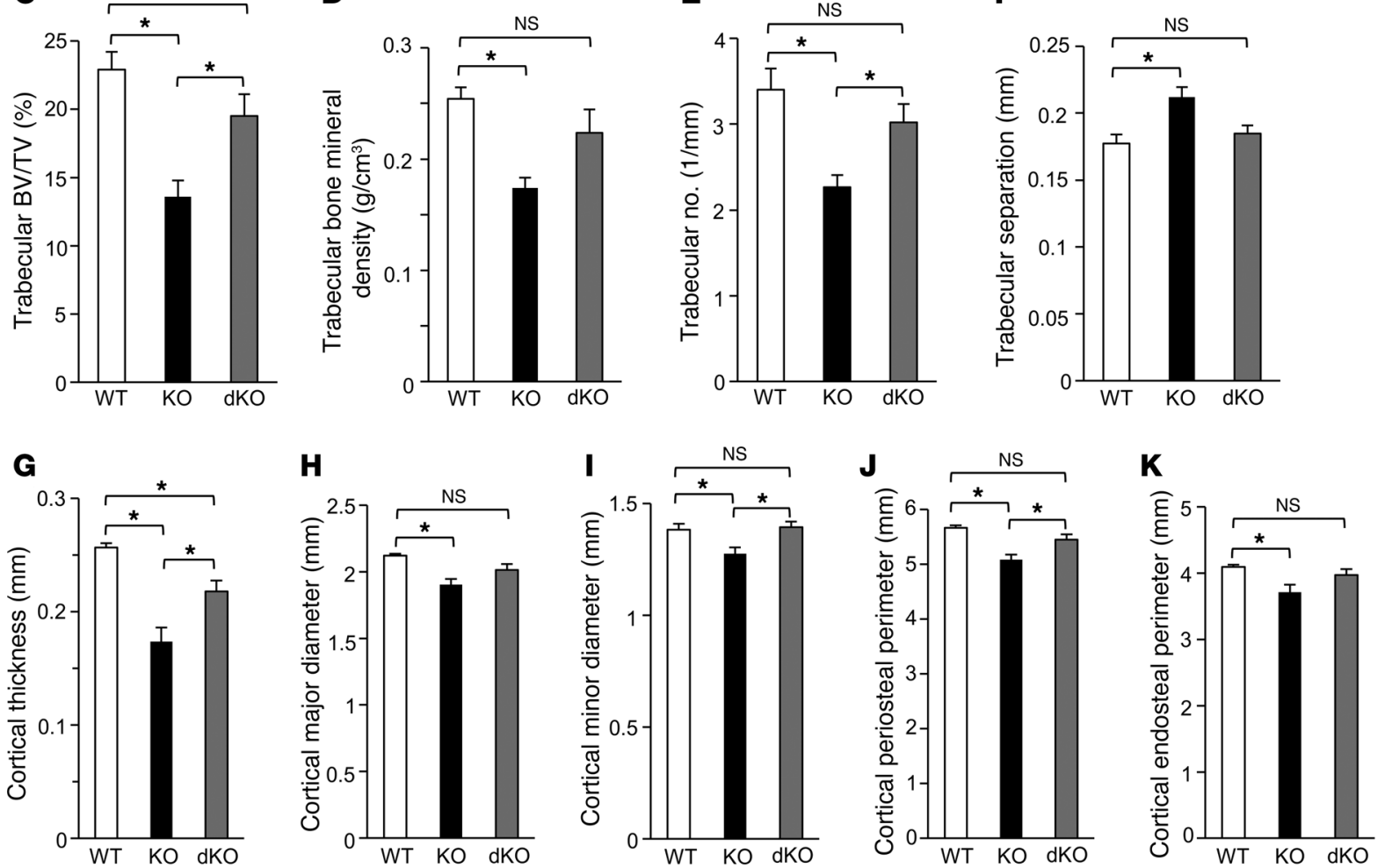

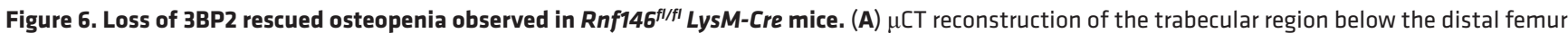
growth plate (top panel) and the cortical region of midshaft femurs (bottom panel) of 12-week-old WT and Sh3bp $2^{f / f l}$ LysM-Cre mice. (B) $\mu C T$ reconstruction of the trabecular region below the distal femur growth plate (top panel) and the cortical region of midshaft femurs (bottom panel) of 12-week-old WT,

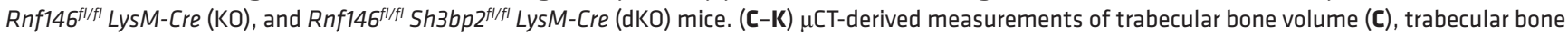
mineral density $(\mathbf{D})$, trabecular number $(\mathbf{E})$, trabecular separation $(\mathbf{F})$, cortical thickness $(\mathbf{G})$, cortical major diameter $(\mathbf{H})$, cortical minor diameter $(\mathbf{I})$, cortical periosteal perimeter (J) and cortical endosteal perimeter (K) of 12-week-old WT, Rnf146 $6^{f / f l}$ LysM-Cre (KO), and Rnf146 fl/fl Sh3bp $2^{f / / f l}$ LysM-Cre (dKO) mice. $n=6$. $P$ values were determined by ANOVA with Tukey-Kramer's post hoc test. Data are presented as mean \pm SEM. ${ }^{*} P<0.05$.

LysM-Cre mice was rescued in Rnf146 $6^{f / f l} S h 3 b p 2^{f / f l}$ LysM-Cre mice (Figure 6B). Moreover, reductions of trabecular bone volume, trabecular bone mineral density, trabecular number, cortical thickness, cortical major and minor diameter, cortical periosteal and endosteal perimeter, and increased trabecular separation detected in $R n f 146^{f / f l}$ LysM-Cre mice were also rescued in $R n f 146^{f / f l}$ Sh3bp $2^{f / f l} L y s M-C r e$ mice (Figure 6, C-K). These data demonstrate that low bone mass observed in Rnf146 $6^{f / f l} L y s M$-Cre mice was contingent on stabilization of 3BP2 in osteoclast progenitors.

The Wnt/ $\beta$-catenin pathway is impaired in Rnf146 $6^{f / f l}$ LysM-Cre osteoclast progenitors. We next investigated the ramification of RNF146 repression by RANKL on regulating the Wnt/ $\beta$-catenin pathway. We examined the expression levels of AXIN1 in Rnf$146^{f / f l}$ LysM-Cre bone marrow-derived osteoclast progenitors and 
A

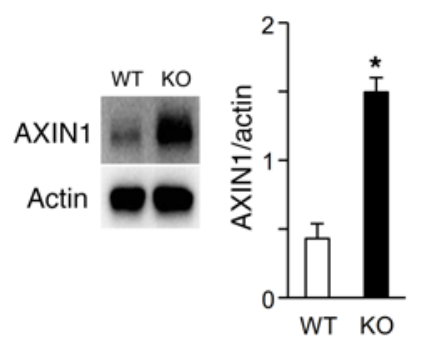

B
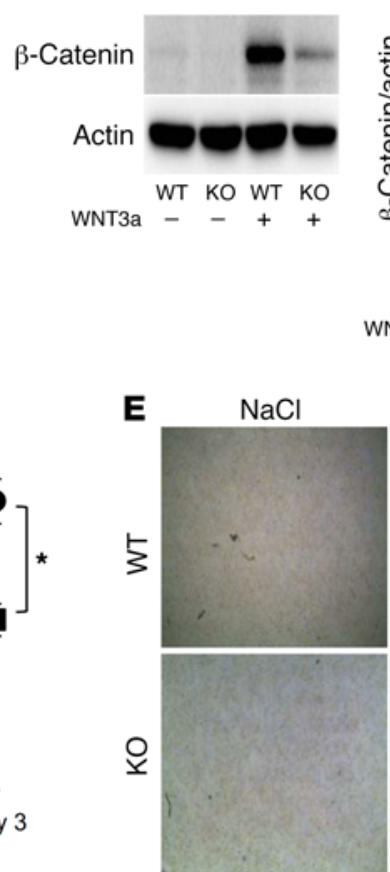
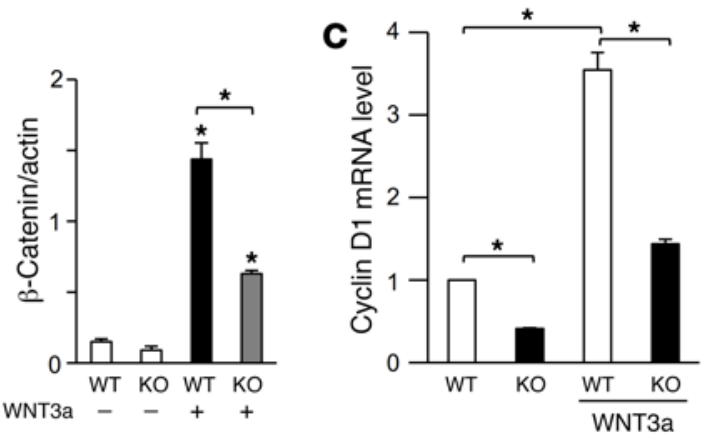

D

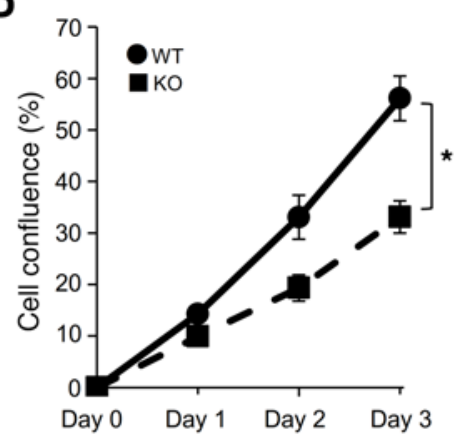

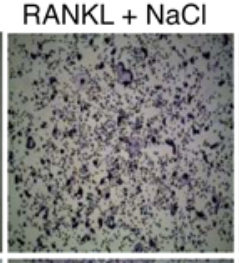

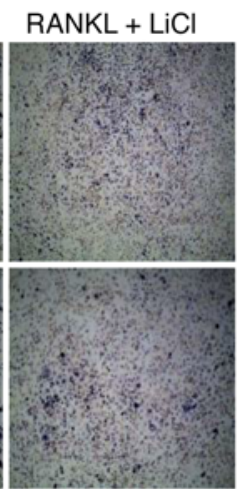

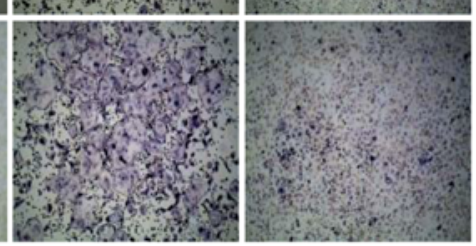

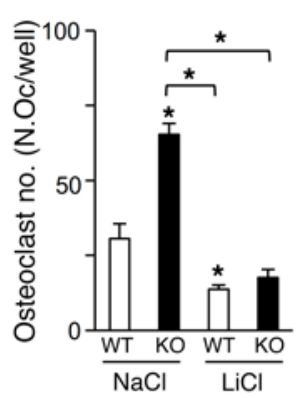

$\mathbf{F}$

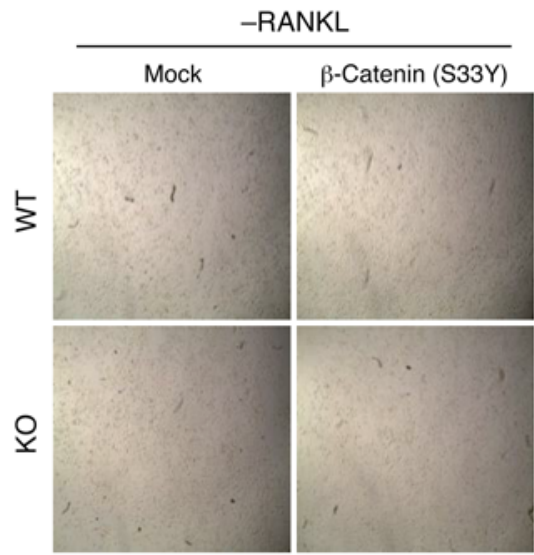

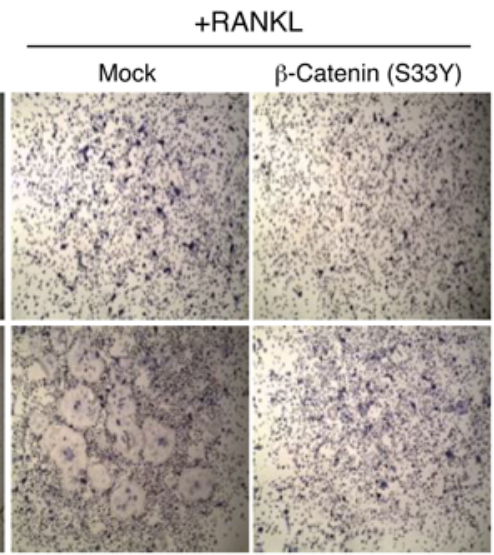

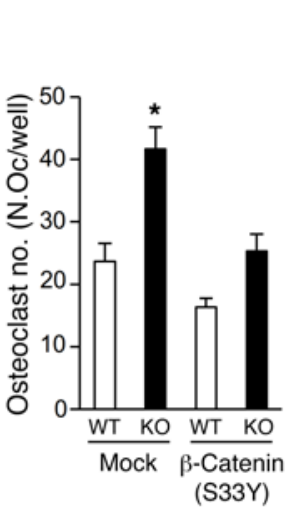

Figure 7. The Wnt/B-catenin pathway is impaired in $R n f 146^{f l / f l}$ LysM-Cre osteoclast progenitors. (A) Whole cell lysates from primary murine osteoclast progenitors derived from WT and Rnf146 fl/fl LysM-Cre (KO) mice were probed with the indicated antibodies for Western blot analysis. (B) Whole cell lysates from cells in A cultured in the presence or absence of a WNT3a-conditioned medium were probed with the indicated antibodies for Western blot analysis. (C) qPCR analysis of cyclin D1 mRNA expression in cells in B. $n=3$. (D) Growth curves of primary murine osteoclast progenitors derived from WT and $R n f 146^{f / f f l}$ LysM-Cre (KO) mice cultured in a WNT3a-conditioned medium for 3 days. $n=3$. (E) TRAP staining of osteoclasts derived from WT and Rnf146 $6^{f / f f}$ LysM-Cre (KO) osteoclast progenitors cultured in the presence or absence of RANKL, NaCl (10 mM), and LiCl (10 mM) for 7 days. Original magnification, $\times 40$. $n=3$. (F) TRAP staining of osteoclasts derived from WT and Rnf146 fl/fl LysM-Cre (KO) osteoclast progenitors infected with an empty vector control (mock) or $\beta$-catenin (S33Y)-expressing retroviral vector and cultured in the presence or absence of RANKL for 7 days. Original magnification, $\times 40$. $n=3$. $P$ values were determined by ANOVA with Tukey-Kramer's post hoc test (B, C, E, and $\mathbf{F})$ or unpaired $t$ test $(\mathbf{A}$ and $\mathbf{D})$. Data are presented as mean $\pm S E M$. ${ }^{*} P<0.05$.

observed that AXIN1 levels were significantly increased (Figure 7A), leading to antagonization of WNT3A-induced $\beta$-catenin stabilization (Figure 7B) and diminished expression of the $\beta$-catenin target gene cyclin D1 (Figure 7C and ref. 23). Consistent with these

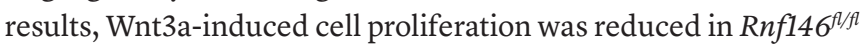
LysM-Cre progenitors compared with that in WT cells (Figure 7D). We hypothesized that loss of RNF146 accelerated the osteoclast developmental program through stabilization of AXIN1 and subsequent impairment of the Wnt/ $\beta$-catenin pathway and queried

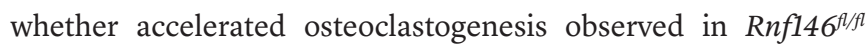
$L y s M$-Cre osteoclast progenitors was restored by activation of the Wnt/ $\beta$-catenin pathway. Inhibition of GSK-3 $\beta$ by LiCl reversed the enhanced osteoclastogenic phenotype observed in Rnf146/ffl LysM-Cre progenitors (Figure 7E and Supplemental Figure 7A). Similarly, ectopic expression of a constitutively active $\beta$-catenin (S33Y) mutant blocked the hyperactivation of osteoclasts observed in RNF146-null progenitors (Figure 7F and Supplemental Figure $7 \mathrm{~B}$ ), indicating that active osteoclastogenesis observed in $R n f 146^{\mathrm{A} / \mathrm{fl}}$ 


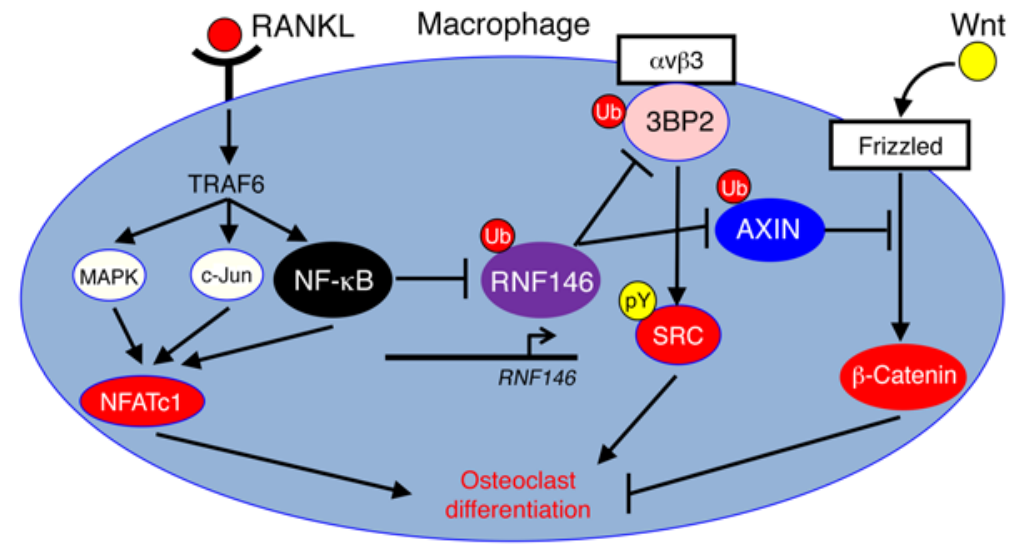

Figure 8. Schematic model showing that RANKL integrates multiple pathways controlling osteoclastogenesis through repression of the E3 ubiquitin ligase RNF146.

RNF146 leads to decreased macrophage proliferation and increased osteoclastogenesis with attenuated $\beta$-catenin expression expands the concept that repression of RNF146 mediates the RANKL-triggered proliferation-to-differentiation switch during osteoclastogenesis.

The 3BP2 degradation program controlled by RNF146 is essential for postnatal skeletal remodeling and TNF- $\alpha$ production. RNF146 is recruited to its substrates through its WWE domain, and its E3 ubiquitin ligase activity is triggered by binding to sites of poly(ADPribose) modification. To date, all known RNF146 substrates are ADP ribosylated by tankyrase. $3 \mathrm{BP} 2$ is a substrate of both tankyrase and RNF146 $(15,21) .3 \mathrm{BP} 2$ missense mutations, associated with the craniofacial dysmorphia syndrome cherubism, uncouple 3BP2 from tankyrase and block 3BP2 ADP ribosylation and subsequent ubiquitylation by RNF146 (15). A knockin

LysM-Cre progenitors is in part through AXIN1-mediated inhibition of the Wnt/ $\beta$-catenin pathway.

These findings conclusively demonstrate that repression of RNF146 by RANKL and subsequent stabilization of 3BP2 and AXIN1 are part of a common genetic pathway required for the osteoclast developmental program.

\section{Discussion}

$R A N K L$ integrates the $3 B P 2$ and $\beta$-catenin signaling pathways required for osteoclastogenesis by transcriptional repression of the E3 ubiquitin ligase RNF146. Osteoclastogenesis is a dynamic process that relies on the activation of distinct signaling pathways that couple to NFATc1, NF-кB, MAPK (24), 3BP2 (14), SRC, VAV, and SYK and is dependent on the simultaneous repression of $\beta$-catenin (11-13). Our findings reveal an unrecognized function of RANKL, coordinating activation of SRC through induction of 3BP2 and repression of $\beta$-catenin together with the canonical RANK signaling pathways required for osteoclastogenesis. Specifically, we found a new role of NF- $\mathrm{BB}$ as a transcriptional repressor of RNF146 (25-27). A reduction of RNF146 expression by NF- $\kappa$ B attenuates ubiquitylation and degradation of its substrates, 3BP2 and AXIN1, leading to activation of SRC kinase and suppression of $\beta$-catenin, respectively (Figure 8). We found that ectopic expression of RNF146 in primary murine macrophages was sufficient to disrupt RANKLmediated stabilization of 3BP2 and AXIN1 and blocked osteoclast differentiation, indicating that modulation of RNF146 expression is a critical control point for the osteoclast developmental program. We have demonstrated that Rnf146 LysM-Cre mice showed severe osteopenia due to hyperactivation of osteoclasts as a consequence of stabilization of 3BP2 and impairment of the Wnt/ $\beta$-catenin pathway. Moreover, deletion of 3BP2 or ectopic expression of active $\beta$-catenin rescued the accelerated osteoclastogenic phenotype observed in RNF146-null osteoclast progenitors. These data demonstrate that loss of RNF146-induced osteoclastogenesis is contingent on activation of the 3BP2/SRC pathway and simultaneous repression of the $\mathrm{Wnt} / \beta$-catenin pathway.

During the process of osteoclast formation, M-CSF promotes monocyte/macrophage proliferation, while RANKL induces osteoclast differentiation $(28,29)$. Our observation that loss of mouse model expressing one of the cherubism-associated mutations in Sh3bp2 displays osteopenia due to active osteoclastogenesis, and bone marrow-derived macrophages from cherubism mice are more susceptive to LPS-induced TNF- $\alpha$ production $(18,30)$. We have shown that $R n f 146^{f l / f l} L y s M$-Cre mice phenocopy cherubism knockin mice with osteopenia and highly activated osteoclasts and that depletion of 3BP2 in Rnf146/f/fl LysM-Cre mice restores this phenotype, showing that the 3BP2 degradation program controlled by RNF146 is critical for optimal postnatal skeletal remodeling.

In addition to its role in osteoclastogenesis, we found that RNF146 is a negative regulator of LPS-induced TNF- $\alpha$ production. Hyperresponsive TLR signaling to commensal bacteria may be an early trigger for autoinflammatory diseases such as rheumatoid arthritis, inflammatory bowel diseases, and periodontitis (31-37). These studies suggest 3BP2 and RNF146 as potential human autoinflammatory disease susceptibility genes. Taken together, these data show that RNF146 acts as an inhibitory switch controlling osteoclastogenesis and cytokine production.

RNF146 is a new target for RANKL. In a recent study, J. Luo et al. demonstrated that leucine-rich repeat-containing $G$ proteincoupled receptor 4 (LGR4) is a new receptor for RANKL (38). They showed that the RANKL/LGR4 signaling pathway inhibits the expression and activity of NFATc1 through activation of Gaq, leading to impairment of osteoclastogenesis, while $\mathrm{Lgrt}^{-/-}$mice suffer from osteopenia due to activation of the osteoclast developmental program (38). These results indicate that RANKL provides a dichotomous signal mediated through 2 distinct receptor systems to control osteoclastogenesis. In addition to the developmental effects of RANKL on osteoclastogenesis, lymph node and lymphocyte development, and breast epithelial proliferation (1-6), recent studies show a link between RANKL and cancer development. The RANKL/RANK pathway mediates breast cancer driven by progestin, which is commonly used for hormone replacement therapy and contraceptives (39). Moreover, RANKL is involved in the progression of osteosarcoma caused by mutations in retinoblastoma (RB) and tumor protein p53 (TP53) tumor suppressors (40), demonstrating that blockade of RANKL could be a therapeutic target for cancer development and progression. Our observation that an interplay between RNF146 and RANKL regulates 
osteoclastogenesis thus suggests insights into the role of RNF146 for cancer biology though the 3BP2/SRC and Wnt/ $\beta$-catenin pathways. Further studies will be necessary to investigate RNF146 in multiple lineages and physiologic states.

\section{Methods}

Generation of Rnf146 conditional knockout mice. In the targeting vector, the first loxP site was inserted in intron 3 , while the second loxP site was placed in the $3^{\prime}$ UTR of exon 4 . Essentially, almost all of the coding region of Rnf146 was flanked by 2 loxP sites. The HSVtk-Neo cassette was flanked by 2 FRT sites. Linearized targeting vector DNA was electroporated into C57BL6/J embryonic stem (ES) cells, and G418-resistant ES clones were first screened by nested PCR and then subjected to Southern blot analysis. Targeted ES clones were used for blastocyst injection, and chimeric males were mated with WT C57BL/6J females to obtain F1 mice. The removal of the HSVtk-Neo cassette was achieved by mating the F1 heterozygous mice with FLPe deleter mice on a C57BL/6J background. Rnf146 $6^{f / f l}$ mice were crossed with LysM-Cre mice (The Jackson Laboratory) (41).

Generation of Sh3bp2 conditional knockout mice. Sh3bp2 exons

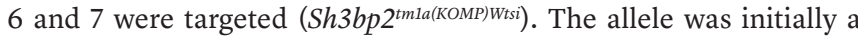
knockout due to the presence of a strong splice acceptor-lacZ-Neo cassette inserted before exon 6. Heterozygous F1 mice with this allele were crossed with actin-FLPe mice (The Jackson Laboratory). FLPe deletes the splice acceptor cassette and generates an allele in which exons 6 and 7 are flanked by loxP sites. Fully verified targeted ES cells were obtained from the Wellcome Trust Sanger Institute Mouse Genetics Project (Sanger MGP). The mouse strain was generated at The Centre for Phenogenomics using the ES cell clone EPD0129_3_CO5 from the Knockout Mouse Project (KOMP) repository at UCD (Davis, California, USA) (project ID CSD39313). Chimeric mice were generated by diploid aggregation of ES cells, and ICR embryos and heterozygous F1 mice harboring a targeted Sh3bp2 allele were obtained by crossing chimeric males with C57BL/6 female mice. Sh3bp $2^{f l / f l}$ mice were crossed with LysM-Cre mice.

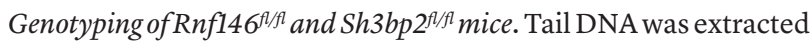
using a Wizard SV Genomic DNA Purification System (Promega), and PCR was performed using GoTaq Green Master Mix (Promega). Floxed alleles were genotyped by PCR using the following primers: Rnf146 (forward primer, 5'-CCAGTCAGACCGATCAGGAACTGAC-3'; reverse primer, 5'-TACTGAACAGCTCTCAACTATCAACACC-3') and Sh3bp2 (forward primer, 5'-ACAGCTCAGTGTTGGATTCCTGGCTC-3'; reverse primer, 5'-ACCCTGACTGGTCGGTGTTTCAGAAC-3').

Cell cultures. HEK293T cells (ATCC) and RAW264.7 cells (ATCC) were cultured in DMEM (Wisent) supplemented with $10 \%$ FBS (Wisent). Bone marrow-derived macrophages were cultured as described previously (15). All cultures were maintained in a $5 \% \mathrm{CO}_{2}$ environment at $37^{\circ} \mathrm{C}$.

Osteoclast differentiation assay. Bone marrow cells were cultured in $\alpha$-MEM (Gibco, Thermo Fisher Scientific) supplemented with $10 \%$ FBS and 2\% CMG (conditioned medium supernatant containing recombinant M-CSF, ref. 42). Osteoclast progenitors were then replated and cultured with recombinant mouse RANKL (10 or $50 \mathrm{ng} / \mathrm{ml}$ ) for 7 days. TRAP staining was performed using a kit from Sigma-Aldrich according to the manufacturer's protocol. Osteoclast numbers were quantified and are shown in the figures as described previously (14). Bone resorption pit assay was performed using Corning Osteo Assay
Surface Plates according to the manufacturer's protocol. Resorption area was quantified and is shown in the figures.

$\mu C T$. Femurs from 12-week-old male mice were scanned using $\mu \mathrm{CT}$ (SkyScan 1174, Bruker) at a resolution of $9.1 \mu \mathrm{m}$ as described previously $(14,15)$. Morphometric analysis was performed on a VOI of trabecular bone $1 \mathrm{~mm}$ in length beginning $1 \mathrm{~mm}$ beneath the first instance of the formation of the mineralized cartilage bridge in the developing metaphysis and on a VOI of cortical bone $0.9 \mathrm{~mm}$ in length in the midshaft. 3D images were reconstructed from $0.5-\mathrm{mm}$ regions within the trabecular and cortical VOIs identified above. Trabecular and cortical morphometric parameters were determined according to standard protocols (43). Trabecular bone mineral density was determined by comparing against a pair of calibration phantoms (SkyScan).

Histomorphometry. In vivo osteoblast parameters were generated from undecalcified tibial sections embedded in methyl methacrylate and stained with Goldner's trichrome. For dynamic histomorphometric analysis, calcein green was injected on days 0 and 7 , and mice were sacrificed 3 days after the second calcein injection. In vivo osteoclast parameters were generated from decalcified tibial sections embedded in paraffin and stained for TRAP activity. Images were analyzed using BIOQUANT software (BIOQUANT Image Analysis Corp.).

In vivo LPS challenge. LPS (Sigma-Aldrich) was injected i.p. at a concentration of $1.8 \mathrm{mg} / \mathrm{kg}$. Blood was harvested 4 hours later by cardiac puncture, and TNF- $\alpha$ in the serum was measured by ELISA (eBioscience) as described previously (30).

Construction and expression of $\beta$-catenin (S33Y) and RNF146 retroviral vectors. $\mathrm{pMXs}-\beta$-catenin (S33Y) was a gift from Shinya Yamanaka (Department of Life Science Frontiers, Center for iPS Cell Research and Application, Kyoto University, Kyoto, Japan) (44). RNF146 cDNA was purchased from Origene Technologies, and pMXs-RNF146 was constructed as described previously (14). HEK293T cells were cotransfected with an empty vector control (mock), pMXs- $\beta$-catenin (S33Y), or pMXs-RNF146 with pMCV-Ecopac (EcoPak) using a CalPhos Mammalian Transfection Kit (Clontech). The virus was collected 48 hours after transfection, and primary murine macrophages were infected and cultured in the presence of RANKL for osteoclast differentiation assay.

In vivo ubiquitin assay. An in vivo ubiquitin assay was performed as described previously $(45,46)$. Briefly, cells cultured in the presence or absence of RANKL (50 ng/ml) were treated with $10 \mu \mathrm{M}$ MG132 for 4 hours prior to collection of cell lysates. Then cells were lysed in complete cell lysis buffer ( $2 \%$ SDS, $150 \mathrm{mM} \mathrm{NaCl}, 10 \mathrm{mM}$ Tris- $\mathrm{HCl}, \mathrm{pH}$ 8.0) with protease and phosphatase inhibitors, boiled for 10 minutes, sheared with a sonication device, and incubated for 30 minutes at $4^{\circ} \mathrm{C}$ with dilution buffer (10 mM Tris- $\mathrm{HCl}, \mathrm{pH} 8.0,150 \mathrm{mM} \mathrm{NaCl}, 2 \mathrm{mM}$ EDTA, 1\% Triton X-100). After lysates had been cleared by centrifugation at $14,000 \mathrm{~g}$ for 30 minutes at $4^{\circ} \mathrm{C}$, immunoprecipitation and Western blotting were performed.

Reagents and antibodies. Unless stated otherwise, all chemicals were purchased from Sigma-Aldrich. Antibodies were obtained from the following sources: anti-pSRC (Y416) (catalog 2101), anti-SRC (catalog 2109), anti-K48 linkage-specific polyubiquitin (catalog 4289), anti-AXIN1 (catalog 2087), anti- $\beta$-catenin (catalog 9562), antiGAPDH (catalog 2118) (Cell Signaling Technology), anti-RNF146 (catalog SAB1408054), anti-Flag M2 (catalog F3165) (SigmaAldrich), anti-actin (catalog sc-47778) (Santa Cruz Biotechnology Inc.), anti-poly(ADP-ribosylation) (anti-PAR) (catalog 4336-APC050) (Trevigen), and anti-3BP2 (catalog H00006452-M01) (Abno- 
va) antibodies. Halt Protease and Phosphatase Inhibitor Cocktail was from Thermo Fisher Scientific.

Promoter assays. HEK293T cells or RAW264.7 cells were transiently cotransfected with the indicated RNF146 promoter constructs and phRL-CMV vector (Promega) using Lipofectamine 2000 (Invitrogen) or Lipofectamine 3000 (Invitrogen), respectively. Cells were lysed and assayed for firefly and renilla luciferase activities using the Dual-Luciferase Reporter System (Promega). Data were expressed as the ratio of firefly activity to renilla activity.

RNA extraction and quantitative real-time PCR analysis. Total cellular RNA was extracted using an RNeasy Plus Mini Kit (QIAGEN). An ImPromII Reverse Transcription System (Promega) was used for reverse transcription, and quantitative real-time PCR (qPCR) was performed on a Step One Plus Real-Time PCR System (Applied Biosystems) using the TaqMan Gene Expression assays (Applied Biosystems) for Gapdh (Mn99999915 g1), Rnf146 (Mn00509629_m1), and Sh3bp2 (Mn00449397_m1). The sequences of primers were as follows: Gapdh (forward primer, $5^{\prime}$-TGTGTCCGTCGTGGATCTG-3'; reverse primer, 5'-GATGCCTGCTTCACCACCTT-3'), Axin1 (forward primer, 5'-CCTCTACCTCACATTCCTCGCACTT-3'; reverse primer, 5'-TCAACCGTTCCTCCAACTTTTCT-3'), Ctsk (forward primer, 5'-CTTGTGGACTGTGTGACTGAG-3'; reverse primer, 5'-CTGGAAAGATGCCAAGCTTGC-3'), Calcr (forward primer, 5'-TTGAGGTTGTGCCCAATGGAG-3'; reverse primer, 5'-GTATGAAGATAGATCCCCTCGC-3'), Acp5 (forward primer, 5'-GCCGGCCACTACCCCATCTGG-3'; reverse primer, 5'-GAGGGATCCATGAAGTTGCCGG-3'), Tnf $\alpha$ (forward primer, 5'-TTGACCTCAGCGCTGAGTTG-3'; reverse primer, 5'-CCTGTAGCCCACGTCGTAGC-3'), and cyclin D1 (forward primer, 5'-AGGCTACAGAAGAGTATTTATGGGAAA-3'; reverse primer, 5'-TGCGTTTGAATCAAGGGAGAT-3'). The relative expression of each mRNA normalized by the expression of Gapdh was calculated by the $\triangle$ Ct method.

ChIP. Endogenous NF- $\mathrm{B}$ was immunoprecipitated from RAW264.7 cell lysates using a SimpleChiP Enzymatic Chromatin IP kit (Cell Signaling Technology, catalog 9003) according to the manufacturer's protocol. Immunoprecipitations were performed with antiNF- $\kappa B$ antibody (catalog 8242, Cell Signaling Technology) or control

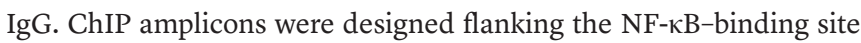
within the Rnf146 promoter. The sequences of primers were as follows: forward primer, 5'-AAATTGGAGCATCTCGGGGA-3'; reverse primer, 5'-TATGTTTCCTTTGGCCGTGC-3'.

Western blot analysis and coimmunoprecipitation. Cells were lysed with RIPA buffer (50 mM Tris [pH 7.5], $150 \mathrm{mM} \mathrm{NaCl,} \mathrm{1 \%} \mathrm{NP40,} \mathrm{0.1 \%}$
SDS, $0.25 \%$ sodium deoxycholate, $1 \mathrm{mM}$ EDTA) supplemented with protease and phosphatase inhibitors as described previously (46). Lysates were cleared by centrifugation at $18,000 \mathrm{~g}$ for 10 minutes at $4^{\circ} \mathrm{C}$. For immunoprecipitation, the products were collected on protein $\mathrm{A}$ or G (Life Technologies). For Western blotting, the protein was resolved by SDS-PAGE and transferred to a PVDF membrane (Immobilon, Millipore). Membranes were blocked in 5\% BSA or 5\% nonfat dried milk in PBST (PBS + 0.1\% Tween-20). The images are representative of 3 independent experiments. The relative integrated densities of each protein band was digitized by Image $(\mathrm{NIH})$ and are shown in the figures.

Cell growth assays. Primary murine osteoclast progenitors were cultured in WNT3a-conditioned medium and placed into the INCUCYTE Kinetic Imaging System (Essen Bioscience) to monitor cell growth and percentage of cell confluence as described previously (47).

Statistics. All results are shown as mean \pm SEM of data from at least 3 separate experiments. Data were subjected to ANOVA with Tukey-Kramer's post hoc test or unpaired $t$ test with JMP 7 (SAS Institute Inc.) to determine differences. $P<0.05$ was considered significant.

Study approval. All animal studies were approved by the Animal Research Council at University Health Network.

\section{Author contributions}

YM and RR designed the experiments. YM performed experiments and analyzed the results. JL, OAK, ML, AC, LZ, YS, MDG, $\mathrm{XM}$, and FC performed specific experiments and analyzed the results. XM and FC contributed materials. YM and RR wrote the manuscript, with helpful comments from OAK.

\section{Acknowledgments}

This work was supported by grants from the Canadian Institute for Health Research (CIHR) (grant 143420). RR is supported by the Princess Margaret Cancer Center and the Ontario Institute for Cancer Research (OICR): ITV. YM is supported by the Postdoctoral Fellowship for Research Abroad contributed by the Japan Society for the Promotion of Science, the Japan Rheumatism Foundation, the Sumitomo Life Social Welfare Services Foundation, and the Nakayama Science Foundation.

Address correspondence to: Robert Rottapel, University Health Network, 101 College Street, TMDT, Room 12-704, Toronto, Ontario, M5G 1L7, Canada. Phone: 416.581.7852; E-mail: rottapel@uhnresearch.ca.
1. Mueller CG, Hess E. Emerging functions of RANKL in lymphoid tissues. Front Immunol. 2012;3:261.

2. Dougall WC, et al. RANK is essential for osteoclast and lymph node development. Genes Dev. 1999;13(18):2412-2424.

3. Kong YY, et al. OPGL is a key regulator of osteoclastogenesis, lymphocyte development and lymph-node organogenesis. Nature. 1999;397(6717):315-323.

4. Kim D, et al. Regulation of peripheral lymph node genesis by the tumor necrosis factor family member TRANCE. JExp Med. 2000;192(10):1467-1478.

5. Knoop KA, Butler BR, Kumar N, Newberry RD, Williams IR. Distinct developmental requirements for isolated lymphoid follicle formation in the small and large intestine: RANKL is essential only in the small intestine. Am J Pathol. 2011;179(4):1861-1871.

6. Perlot T, Penninger JM. Development and function of murine B cells lacking RANK. J Immunol. 2012;188(3):1201-1205.

7. Wiktor-Jedrzejczak W, Bartocci A, Ferrante AW Jr, Ahmed-Ansari A, Sell KW, Pollard JW, Stanley ER. Total absence of colony-stimulating factor 1 in the macrophage-deficient osteopetrotic (op/op) mouse. Proc Natl Acad Sci U S A. 1990;87(12):4828-4832.

8. Yoshida H, et al. The murine mutation osteopetrosis is in the coding region of the macrophage colony stimulating factor gene. Nature. 1990;345(6274):442-444.
9. Boyce BF, Yoneda T, Lowe C, Soriano P, Mundy GR. Requirement of pp60c-src expression for osteoclasts to form ruffled borders and resorb bone in mice. J Clin Invest. 1992;90(4):1622-1627.

10. Lowe C, Yoneda T, Boyce BF, Chen H, Mundy GR, Soriano P. Osteopetrosis in Src-deficient mice is due to an autonomous defect of osteoclasts. Proc Natl Acad Sci U S A. 1993;90(10):4485-4489.

11. Albers J, et al. Canonical Wnt signaling inhibits osteoclastogenesis independent of osteoprotegerin. J Cell Biol. 2013;200(4):537-549.

12. Otero K, et al. TREM2 and $\beta$-catenin regulate bone homeostasis by controlling the rate of osteoclastogenesis. Jimmunol. 2012;188(6):2612-2621. 
13. Wei $\mathrm{W}$, et al. Biphasic and dosage-dependent regulation of osteoclastogenesis by $\beta$-catenin. $\mathrm{Mol}$ Cell Biol. 2011;31(23):4706-4719.

14. Levaot N, et al. 3BP2-deficient mice are osteoporotic with impaired osteoblast and osteoclast functions. J Clin Invest. 2011;121(8):3244-3257.

15. Levaot N, et al. Loss of Tankyrase-mediated destruction of $3 \mathrm{BP} 2$ is the underlying pathogenic mechanism of cherubism. Cell. 2011;147(6):1324-1339.

16. Jones WA, Gerrie J, Pritchard J. Cherubism familial fibrous dysplasia of the jaws. J Bone Joint Surg Br. 1950;32-B(3):334-347.

17. Ueki Y, et al. Mutations in the gene encoding c-Abl-binding protein SH3BP2 cause cherubism. Nat Genet. 2001;28(2):125-126.

18. Ueki Y, et al. Increased myeloid cell responses to M-CSF and RANKL cause bone loss and inflammation in SH3BP2 "cherubism" mice. Cell. 2007;128(1):71-83.

19. Zeng L, et al. The mouse Fused locus encodes Axin, an inhibitor of the Wnt signaling pathway that regulates embryonic axis formation. Cell. 1997;90(1):181-192.

20. Kikuchi A. Roles of Axin in the Wnt signalling pathway. Cell Signal. 1999;11(11):777-788.

21. Zhang Y, et al. RNF146 is a poly(ADP-ribose)directed E3 ligase that regulates axin degradation and Wnt signalling. Nat Cell Biol. 2011;13(5):623-629.

22. Kel AE, Gössling E, Reuter I, Cheremushkin E, Kel-Margoulis OV, Wingender E. MATCH: A tool for searching transcription factor binding sites in DNA sequences. Nucleic Acids Res. 2003;31(13):3576-3579.

23. Tetsu O, McCormick F. $\beta$-Catenin regulates expression of cyclin D1 in colon carcinoma cells. Nature. 1999;398(6726):422-426.

24. Feng X, Teitelbaum SL. Osteoclasts: new insights. Bone Res. 2013;1(1):11-26.

25. Tordella L, et al. ASPP2 suppresses squamous cell carcinoma via RelA/p65-mediated repression of p63. Proc Natl Acad Sci US A.
2013;110(44):17969-17974.

26. Zhang W, Kone BC. NF- $\mathrm{kB}$ inhibits transcription of the $\mathrm{H}(+)-\mathrm{K}(+)$-ATPase $\alpha(2)$-subunit gene: role of histone deacetylases. Am J Physiol Renal Physiol. 2002;283(5):F904-F911.

27. Datta De D, Datta A, Bhattacharjya S, Roychoudhury S. NF- $\mathrm{kB}$ mediated transcriptional repression of acid modifying hormone gastrin. PLOS One. 2013;8(8):e73409.

28. Mizoguchi T, et al. Identification of cell cyclearrested quiescent osteoclast precursors in vivo. JCell Biol. 2009;184(4):541-554.

29. Sankar U, Patel K, Rosol TJ, Ostrowski MC. RANKL coordinates cell cycle withdrawal and differentiation in osteoclasts through the cyclindependent kinase inhibitors p27KIP1 and p21CIP1 J Bone Miner Res. 2004;19(8):1339-1348.

30. Prod'Homme V, et al. Cherubism allele heterozygosity amplifies microbe-induced inflammatory responses in murine macrophages. J Clin Invest. 2015;125(4):1396-1400.

31. Bain CC, Mowat AM. Macrophages in intestinal homeostasis and inflammation. Immunol Rev. 2014;260(1):102-117.

32. Graves DT, Li J, Cochran DL. Inflammation and uncoupling as mechanisms of periodontal bone loss. J Dent Res. 2011;90 (2):143-153.

33. Caradonna L, Amati L, Magrone T, Pellegrino NM, Jirillo E, Caccavo D. Enteric bacteria, lipopolysaccharides and related cytokines in inflammatory bowel disease: biological and clinical significance. JEndotoxin Res. 2000;6(3):205-214.

34. Rhee SH. Lipopolysaccharide: basic biochemistry, intracellular signaling, and physiological impacts in the gut. Intest Res. 2014;12(2):90-95.

35. Im E, Riegler FM, Pothoulakis C, Rhee SH. Elevated lipopolysaccharide in the colon evokes intestinal inflammation, aggravated in immune modulator-impaired mice. Am J Physiol Gastrointest Liver Physiol. 2012;303(4):G490-G497.

36. Cario E, Podolsky DK. Differential alteration in intestinal epithelial cell expression of toll-like receptor 3 (TLR3) and TLR4 in inflammatory bowel disease. Infect Immun. 2000;68(12):7010-7017.

37. Fort MM, et al. A synthetic TLR4 antagonist has anti-inflammatory effects in two murine models of inflammatory bowel disease. J Immunol. 2005;174(10):6416-6423.

38. Luo J, et al. LGR4 is a receptor for RANKL and negatively regulates osteoclast differentiation and bone resorption. Nat Med.2016;22(5):539-546.

39. Schramek D, et al. Osteoclast differentiation factor RANKL controls development of progestin-driven mammary cancer. Nature. 2010;468(7320):98-102.

40. Chen Y, et al. RANKL blockade prevents and treats aggressive osteosarcomas. Sci Transl Med. 2015;7(317):317ra197.

41. Clausen BE, Burkhardt C, Reith W, Renkawitz $\mathrm{R}$, Förster I. Conditional gene targeting in macrophages and granulocytes using LysMcre mice. Transgenic Res. 1999;8(4):265-277.

42. Takeshita S, Kaji K, Kudo A. Identification and characterization of the new osteoclast progenitor with macrophage phenotypes being able to differentiate into mature osteoclasts. J Bone Miner Res. 2000;15(8):1477-1488.

43. Parfitt AM, et al. Bone histomorphometry: standardization of nomenclature, symbols, and units. Report of the ASBMR Histomorphometry Nomenclature Committee. J Bone Miner Res. 1987;2(6):595-610.

44. Takahashi K, Yamanaka S. Induction of pluripotent stem cells from mouse embryonic and adult fibroblast cultures by defined factors. Cell. 2006;126(4):663-676.

45. Choo YS, Zhang Z. Detection of protein ubiquitination. J Vis Exp. 2009;(30):1293.

46. Matsumoto $Y$, et al. Reciprocal stabilization of ABL and TAZ regulates osteoblastogenesis through transcription factor RUNX2. J Clin Invest. 2016;126(12):4482-4496.

47. Cullis J, et al. The RhoGEF GEF-H1 is required for oncogenic RAS signaling via KSR-1. Cancer Cell. 2014;25(2):181-195 